\title{
A Fully Integrated 24-GHz Phased-Array Transmitter in CMOS
}

\author{
Arun Natarajan, Student Member, IEEE, Abbas Komijani, Student Member, IEEE, and Ali Hajimiri, Member, IEEE
}

\begin{abstract}
This paper presents the first fully integrated 24-GHz phased-array transmitter designed using $0.18-\mu \mathrm{m}$ CMOS transistors. The four-element array includes four on-chip CMOS power amplifiers, with outputs matched to $50 \Omega$, that are each capable of generating up to $14.5 \mathrm{dBm}$ of output power at $24 \mathrm{GHz}$. The heterodyne transmitter has a two-step quadrature up-conversion architecture with local oscillator (LO) frequencies of 4.8 and 19.2 GHz, which are generated by an on-chip frequency synthesizer. Four-bit LO path phase shifting is implemented in each element at 19.2 GHz, and the transmitter achieves a peak-to-null ratio of $23 \mathrm{~dB}$ with raw beam-steering resolution of $7^{\circ}$ for radiation normal to the array. The transmitter can support data rates of $500 \mathrm{Mb} / \mathrm{s}$ on each channel (with BPSK modulation) and occupies $6.8 \mathrm{~mm} \times 2.1 \mathrm{~mm}$ of die area.
\end{abstract}

Index Terms-CMOS, integrated circuits, multiphase oscillator, phased arrays, power amplifiers, radar, transmitter, wireless communications.

\section{INTRODUCTION}

$\mathbf{H}$ IGH-FREQUENCY phased-array systems have been ubiquitous in the fields of radar and radio astronomy [1]-[3]. However, these systems rely on specialized discrete components and careful assembly of modules that increase cost and complexity of manufacturing. Integration on silicon makes it possible to realize complex phased-array systems, with on-chip mixed-signal and digital signal processing, at lower cost and with higher reliability. Furthermore, digital tuning and calibration in integrated systems can be used to improve the performance of critical analog/RF parts [4], [5]. Importantly, the very low incremental cost of signal processing elements, i.e., transistors, and the benefits of integration such as short and robust interconnects and good component matching enable the realization of novel phased-array architectures that are designed specifically for existing and emerging applications [6], [7]. For instance, phased-array-based transmitters and receivers, operating at high frequencies where large bandwidths are available, are well suited for high data rate directional point-to-point wireless communication networks and for short-range radar applications such as collision avoidance and assisted parking in automobiles.

The physical size of such integrated phased-array systems is restricted by the size of the antennas and the spacing between them. In a system employing resonance-based antennas, the antenna size and spacing decrease with an increase in frequency.

Manuscript received April 26, 2005; revised July 15, 2005.

The authors are with the California Institute of Technology, Pasadena, CA 91125 USA (e-mail: arun@caltech.edu).

Digital Object Identifier 10.1109/JSSC.2005.857420
Thus, the large bandwidths and smaller physical system size render high frequencies attractive for integrated phased-array implementation.

A particularly interesting frequency for integrated phasedarray system implementation is $24 \mathrm{GHz}$. The Industrial, Scientific, and Medical (ISM) band at $24 \mathrm{GHz}$ has been opened up for wireless point-to-point communications by the Federal Communications Commission (FCC) [8]. In addition, $7 \mathrm{GHz}$ of spectrum from 22 to $29 \mathrm{GHz}$ has been allocated for ultra-wideband vehicular radar applications, making the 24-GHz band appealing from both wireless communication and car radar perspectives.

CMOS process technologies are the most attractive among silicon-based technologies for integrated systems due to the benefits of scaling and the possibility of integrating the digital backbone with the RF front-end. However, the low active gain of MOS transistors and the lossy passives at high frequencies have prevented any significant movement toward integrating entire high-frequency phased-array systems on CMOS technologies. The fully integrated four-element $24-\mathrm{GHz}$ phased-array transmitter, with on-chip power amplifiers, reported in this paper is not only the first fully integrated phased-array transmitter but also the first system to demonstrate such levels of integration at $24 \mathrm{GHz}$ using $0.18-\mu \mathrm{m} \mathrm{CMOS}$ transistors. The transmitter reported in this paper and the eight-element phased-array SiGe receiver presented in [6] demonstrate the feasibility of $24-\mathrm{GHz}$ phased-array systems in silicon-based processes.

In the following sections, the system level and circuit level aspects in the design of the transmitter will be discussed in depth. Section II provides a brief introduction to the properties and advantages of phased-array transmitters while Section III discusses the tradeoffs of high-frequency phased-array wireless communication. Different phased-array architectures, particularly the local oscillator (LO) phase-shifting architecture adopted in this paper, are examined in Section IV. The architecture of the transmitter and the design of key circuit building blocks are described in Section V, and the measurement results are presented in Section VI.

\section{Phased-ARray Principles}

In a multiple-element transmitter, a beam is formed in a desired direction by varying the relative delay in each element to compensate for the difference in propagation delays for signals from different elements. Electronic variation of the delay enables beam steering without actual mechanical reorientation of the antennas. Fig. 1 shows a simplified $n$-element phased-array transmitter. When the input signal $s(t)$ is distributed to elements 


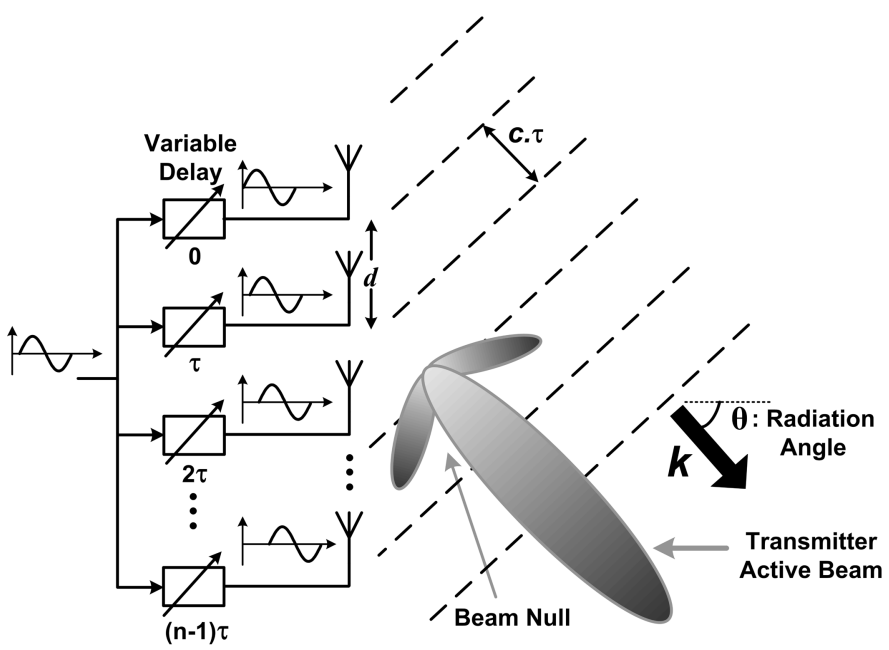

Fig. 1. $n$-element phased-array transmitter.

that delay the signal by multiples of $\tau$, the combined signal in a direction $\theta$ is given by

$$
S(t)=\sum_{k=0}^{n-1} s\left(t-k \tau-(n-1-k) \frac{d \sin \theta}{c}\right) .
$$

Therefore, the signals from all elements add up coherently in the direction $\theta=\sin ^{-1}(c \tau / d)$, where $d$ is the spacing between antennas and $c$ is the velocity of light. This coherent addition increases the power radiated in the desired direction while incoherent addition of the signal in other directions ensures lower interference power at receivers that are not targeted. It can be seen from (1) that in an $n$-element transmitter, if each element radiates $P$ watts omnidirectionally, the effective isotropic radiated power (EIRP) $)^{1}$ in the main beam direction is $n^{2} P$ watts. For example, if each transmitter in a four-element array radiates $14 \mathrm{dBm}$, the EIRP in the beam direction is increased by $12 \mathrm{~dB}\left(20 \log _{10} 4\right)$ to $26 \mathrm{dBm}$. This increase in signal power at the receiver is particularly useful at high frequencies, where the efficiency and output power of silicon-based power amplifiers are low, path loss is high, and the receiver sensitivity is low. From a system perspective, implementing a phased array at the receiver side as well improves receiver sensitivity, thereby increasing channel capacity. The directivity of the phased-array transmitter-receiver system permits higher frequency reuse due to better interference suppression and rejection, leading to increased network capacity.

\section{High-FrEQUENCY WIRELESS COMMUNICATION}

The large bandwidths and smaller physical system size motivate a move to high frequencies for wireless communications. In this section, the tradeoffs of high-frequency wireless networks are discussed, with an emphasis on phased-array systems.

The beamforming and electronic beam-steering properties of phased arrays provide a solution to the demand for directional

\footnotetext{
${ }^{1}$ The EIRP in a particular direction is the power that an isotropic transmitter
} would have to radiate to cause the same field strength in that direction.

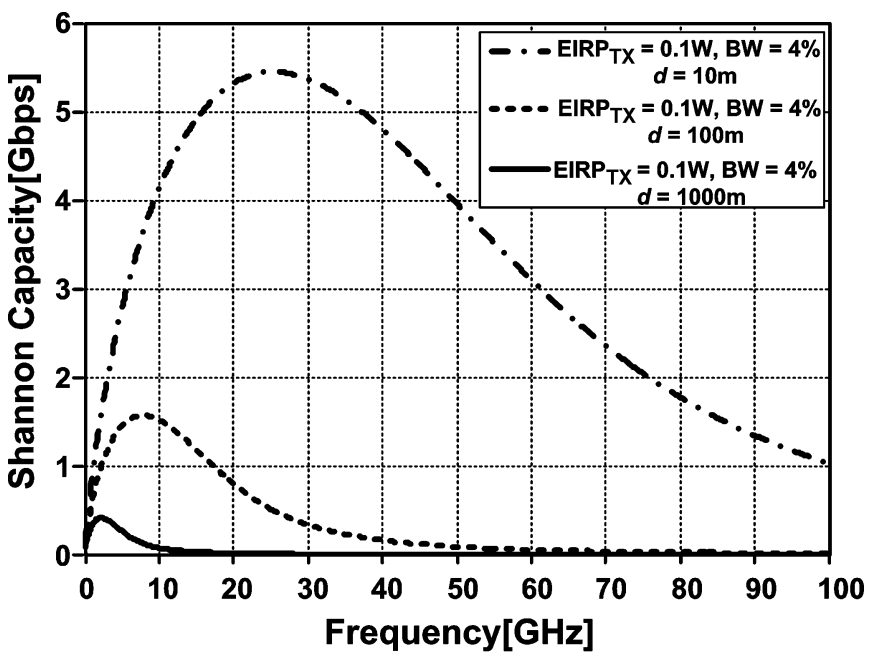

(a)

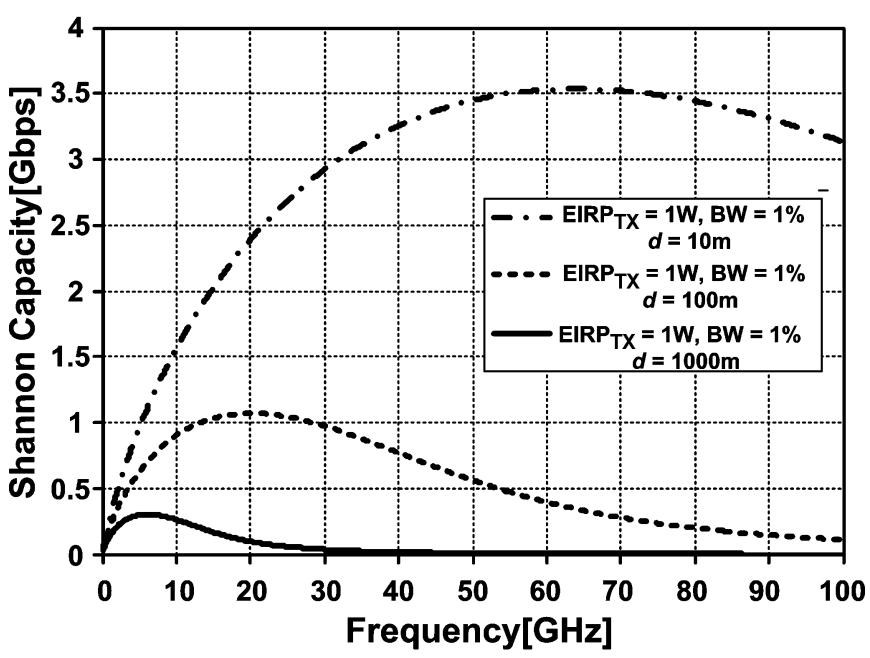

(b)

Fig. 2. Comparison of channel capacities for different system parameters demonstrates the tradeoffs of moving to high frequencies. (a) Channel capacity for EIRP $=0.1 \mathrm{~W}, \mathrm{BW}=4 \%$. (b) Channel capacity for EIRP $=1 \mathrm{~W}$, $\mathrm{BW}=1 \%$.

wireless links at high frequencies. ${ }^{2}$ Resonance-based planar antennas such as dipoles or patch antennas are well suited for an integrated phased-array-based system with electronic beam steering. A typical patch antenna array at $24 \mathrm{GHz}$, for instance, has an antenna gain of $10 \mathrm{~dB}[9] .^{3}$ According to the antenna theorem, the aperture area of an antenna, for a given gain, decreases with an increase in frequency, reducing the power collected at the receiver at high frequencies [10]. The collected power cannot be increased by increasing the aperture area, as that increases antenna gain, making the antenna more directional and hence limiting the angles at which phased-array operation is possible. Thus, in a resonance-antenna-based system,

\footnotetext{
${ }^{2}$ The spatial specifications in the 24- to $24.25-\mathrm{GHz}$ band call for a main lobe beamwidth not exceeding $3.5^{\circ}$ in the azimuthal and the elevation planes [8].

${ }^{3} \mathrm{~A}$ four-element array, implemented with antennas that have 10-dB gain, will have an effective antenna gain of $22 \mathrm{~dB}$. Therefore, in the four-element array, to achieve the maximum permitted EIRP of $29.7 \mathrm{dBm}$ in the $24-$ to $24.25-\mathrm{GHz}$ band [8], each transmitter has to generate $7.7 \mathrm{dBm}$. In the 22- to $29-\mathrm{GHz}$ band, the low EIRP requirements $(\sim-2.5 \mathrm{dBm})$ make large bandwidth the primary focus of design.
} 
the advantage of large bandwidths at high frequencies is offset to some extent by the lower received power and the higher receiver noise figure at high frequencies.

For a transmit EIRP of $P_{\mathrm{TX}}$, the signal power at the receiver $P_{\mathrm{RX}}$ is given by Friis' equation [11]

$$
P_{\mathrm{RX}}=P_{\mathrm{TX}}\left(\frac{\lambda}{4 \pi}\right)^{2} \frac{G_{\mathrm{TX}} G_{\mathrm{RX}}}{d^{l}}
$$

where $G_{\mathrm{TX}}$ and $G_{\mathrm{RX}}$ are the receiver and transmitter antenna gain, $\lambda$ is the wavelength, $d$ is the distance between transmitter and receiver, and $l$ is the exponent that can be higher than the nominal value of 2 to account for excess path loss. In order to explore the tradeoffs of moving to high frequencies, the channel capacity $C$ (in bit per second) given by Shannon's theorem [12]

$$
C=\mathrm{BW} \log _{2}(1+\mathrm{SNR})
$$

is calculated for different carrier frequencies $f_{c}$ while keeping the fractional bandwidth constant, accounting for larger bandwidths at higher frequencies. The noise figure of the receiver is assumed to increase linearly with frequency [13]. A noise figure of $3 \mathrm{~dB}$ is assumed at $6 \mathrm{GHz}$, resulting in a noise figure of $7 \mathrm{~dB}$ at $24 \mathrm{GHz} .{ }^{4}$ The individual antennas are assumed to be isotropic and no excess path loss is considered (i.e., $l=2$ ). Fig. 2(a) and (b) plots the channel capacity against carrier frequencies under the assumptions made above for two sets of EIRP and fractional bandwidth values. The fact that the curves are not monotonic and exhibit a peak demonstrates the tradeoff between larger bandwidths, lower collected power, and higher noise figure at high frequencies. The peak in the channel capacity curve moves to lower frequencies for higher transmitter-receiver separations and moves to higher frequencies with higher transmit EIRP or lower noise figure, both of which can be improved by implementing multiple-antenna systems such as phased arrays. Fig. 3 plots the channel capacity for different transmitter-receiver separations, assuming a bandwidth of $250 \mathrm{MHz}$, EIRP of $30 \mathrm{dBm}$, and receiver noise figure of $7 \mathrm{~dB}$ at $24 \mathrm{GHz}$. In a four-element phased-array receiver, the signal-to-noise ratio (SNR) at the output may be improved by up to $6 \mathrm{~dB}$ [6]. When this improvement in SNR is included in (2) and (3), it can be seen that high-speed (greater than $1 \mathrm{~Gb} / \mathrm{s}$ ) phased-array data links are possible up to a distance of $200 \mathrm{~m}$ at $24 \mathrm{GHz}$.

\section{Phased-ARRAY ARChiteCtURES}

\section{A. Delay and Phase-Shifting Architectures}

Ideally, to achieve broadband phased-array operation, a truetime delay is required in each element. In the architecture in Fig. 4(a), the required time delay is implemented in the RF path in each element. However, implementing a broadband low-loss true-time delay element at RF, which is capable of large variation, occupies a practical area, and scales well with an increase in number of elements, poses several problems. The true-time delay in the RF path can be replaced equivalently by a delay in the IF path and a phase shift in the LO path/IF path as shown in Fig. 4(b), or by implementing the delay in the digital domain

\footnotetext{
${ }^{4}$ Noise figure $F=1+(f / 6 e 9)$.
}

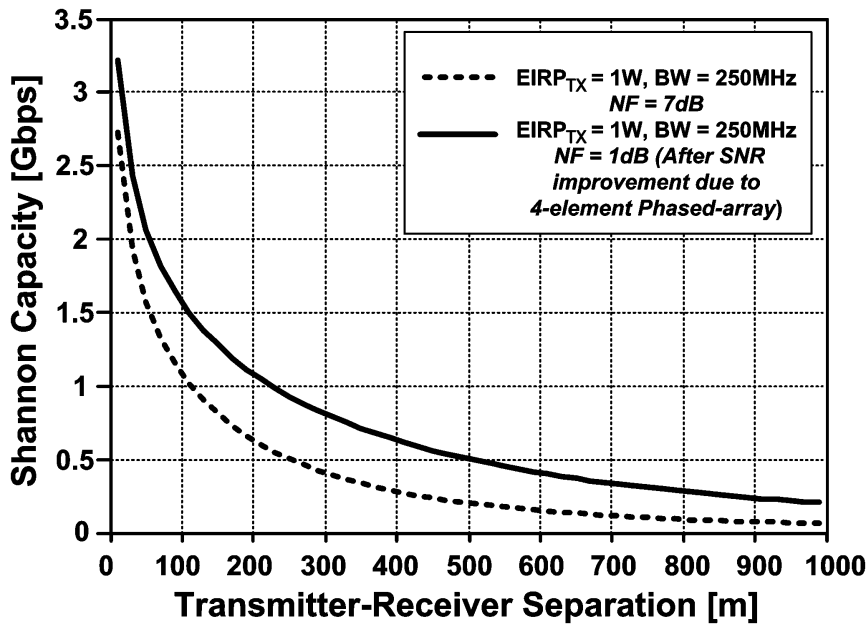

Fig. 3. Channel capacity for different transmitter-receiver separations at $24 \mathrm{GHz}$.

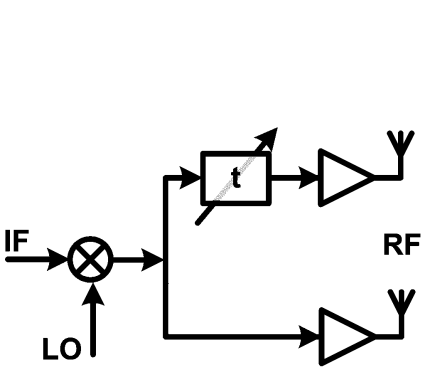

(a)

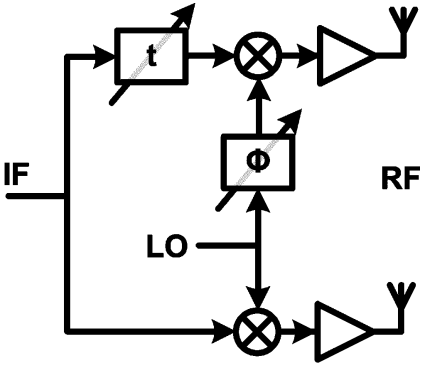

(b)

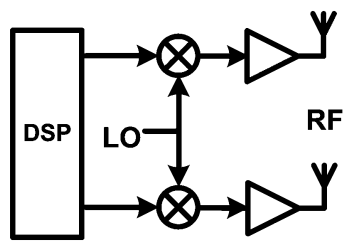

(c)

Fig. 4. Broadband array architectures. (a) Delay in RF path. (b) Delay in IF path and LO phase shift. (c) Digital delay at baseband.

as shown in Fig. 4(c). While an analog delay element at IF has the same problems as a delay at RF, practical considerations of mixed-signal circuits and digital signal processor (DSP) performance limit the digital array architecture [14].

If the bandwidth of interest is sufficiently narrow, the time delay (a linear phase shift in the frequency domain) can be approximated by a constant phase shift at the center frequency. The phase-shift architecture can be implemented as an approximation of the delay-based architecture in Fig. 4(a), which leads to a phase shift in the RF signal path [Fig. 5(a)], or as an approximation of the architecture in Fig. 4(b), which leads to a phase shift in the LO path or IF signal path [Fig. 5(b) and (c)].

Low-loss phase shifters in the RF path have attracted research interest [15], [16]. If the phase-shifter loss is not uniform for all phase shifts, variable gain amplifiers are required in each element to equalize the phase-shifter losses to avoid array pattern degradation.

Phase shifters in the LO path circumvent this problem as the circuits in the LO path such as the voltage-controlled oscillator 

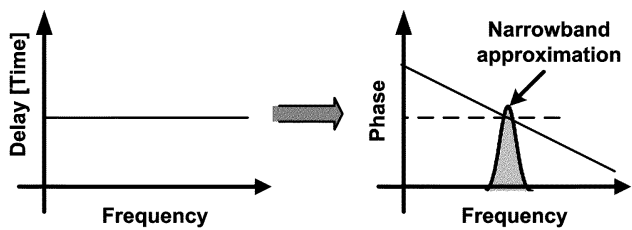

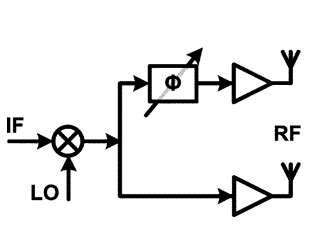

(a)

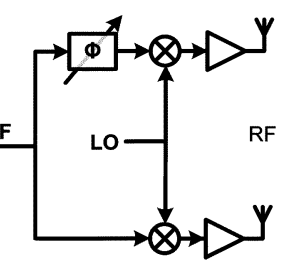

(b)

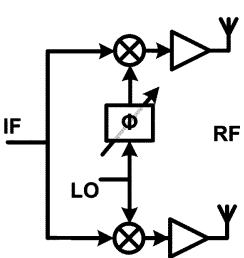

(c)
Fig. 5. Narrowband phased-array architectures. (a) RF phase shift. (b) IF phase shift. (c) LO phase shift.

(VCO) and the LO path amplifiers operate in saturation by design since the performance of the mixers in the up-conversion path is improved with larger LO voltage swings. Furthermore, with large LO signal swings at the LO ports of the mixers, the sensitivity of mixer gain to the LO signal amplitude is low. As a result, with phase shifters in the LO path, the variation in signal amplitude for different values of phase shift is minimal. Therefore, the LO path phase-shifting architecture has been chosen for the phased-array transmitter.

\section{B. Effects of Narrowband Approximation}

The narrowband LO phase-shift architecture [shown in Fig. 5(c)] leads to some signal distortion due to dispersion. The input signal that is distributed to each element in a phased-array transmitter can be represented as $s(t)=v(t) \cos \left[\omega_{\mathrm{RF}} t+\phi(t)\right]$, where $v(t)$ and $\phi(t)$ represent the baseband signal modulating the carrier. When phase shifts are implemented in each element to achieve a radiation angle of $\theta$, for which the propagation delay between successive elements differs by $\tau$, the combined signal power is

$$
S(t)=\sum_{k=0}^{n-1} v(t-k \tau) \cos \left[\omega_{\mathrm{RF}} t+\phi(t-k \tau)\right]
$$

Thus, none of the phase-shifting architectures ensure that the baseband modulating signals add up coherently resulting in a signal distortion that is manifested as a higher error vector magnitude (EVM). EVM increases with an increase in the number of elements and/or the bandwidth of the baseband signal, and can be a source of error on both the transmitter and receiver side leading to higher bit error rates [17]. As will be explained below, a method to mitigate the deterministic errors caused by the phase-shift approximation is to use a normalized orthogonal frequency division multiplexing (OFDM) modulation scheme for the baseband input that is distributed to all the elements in the transmitter.

For a QPSK signal at data rate $R$ bits per second, the time period of each symbol $T_{s}=1 / 2 R$. Therefore, the complex digital baseband signal is $s_{d}\left(n T_{s}\right)=b_{n}$ where $b_{n}$ is complex. In OFDM, the total bandwidth of the system is divided into $N_{c}$

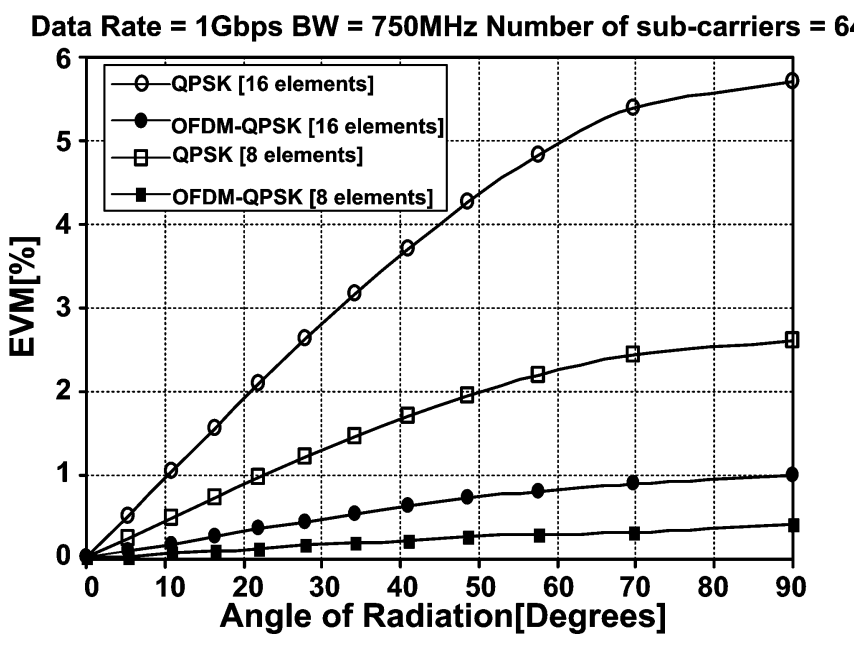

Fig. 6. EVM improvement provided by normalized OFDM-QPSK modulation over QPSK modulation for 8-element and 16-element transmitter.

channels, with the $N_{c}$ subcarriers orthogonal. The baseband digital data are divided into $N_{c}$ parallel streams, each of which is used to modulate a subcarrier [18]. Thus, each OFDM symbol consists of $N_{c}$ QPSK symbols, and for each frame (of duration $\left.N_{c} T_{s}\right), s_{d}\left(n T_{s}\right)=\sum_{k=0}^{N_{c}-1} b_{k} e^{j 2 \pi\left(k n / N_{c}\right)}$

Since each of the channels in the OFDM scheme is narrowband, the error due to the time delay being replaced by a constant phase shift can be largely corrected by multiplying the input modulating each subcarrier by a complex normalizing factor $\alpha_{k}$ such that

$$
s_{d}\left(n T_{s}\right)=\sum_{k=0}^{N_{c}-1} \alpha_{k} b_{k} e^{j 2 \pi \frac{k n}{N_{c}}}
$$

The memoryless predistortion coefficients $\alpha_{k}$ depend upon the direction of radiation that is known a priori in the transmitter. The same normalization scheme can also be implemented in a phased-array receiver to account for similar signal distortion due to dispersion. Fig. 6 compares the EVM for a raw QPSK modulation scheme and for a 64-subcarrier OFDM-QPSK modulation scheme with complex normalization in a $24-\mathrm{GHz}$ phased-array transmitter with 8 and 16 elements. The signal has a data rate of $1 \mathrm{~Gb} / \mathrm{s}$ and a bandwidth of $750 \mathrm{MHz}$. The maximum EVM, corresponding to a worst-case radiation angle of $90^{\circ}$, improves from $5.8 \%$ to $1 \%$ in the 16 element case, demonstrating the efficacy of the normalized OFDM scheme. Thus, careful choice of modulation schemes and simple equalization methods can render the implementation of actual analog or digital delay in each element unnecessary. Increasing the number of subcarriers decreases the EVM further and can compensate for increased distortion due to higher signal bandwidth and/or higher number of elements in the array.

\section{Array Pattern Performance}

The transmitter described in this paper is based on a phaseshifting architecture that was introduced in [14] in the context of a receiver. Fig. 7(a) shows a simplified four-element phasedarray transmitter with LO path phase shifting. Relative phase 


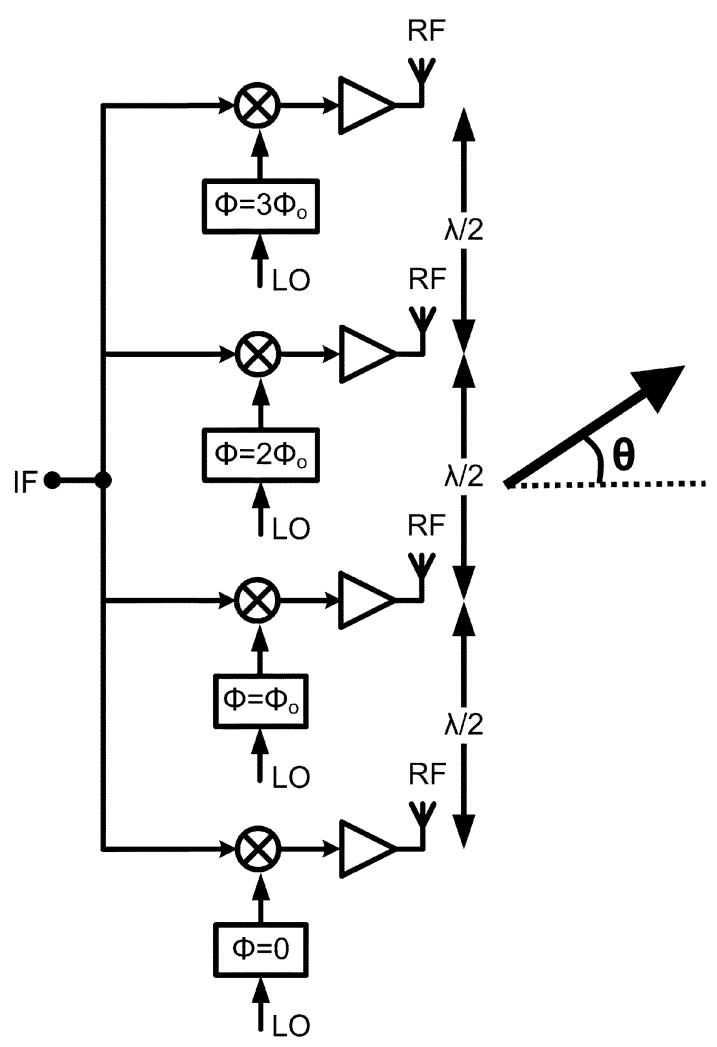

(a)

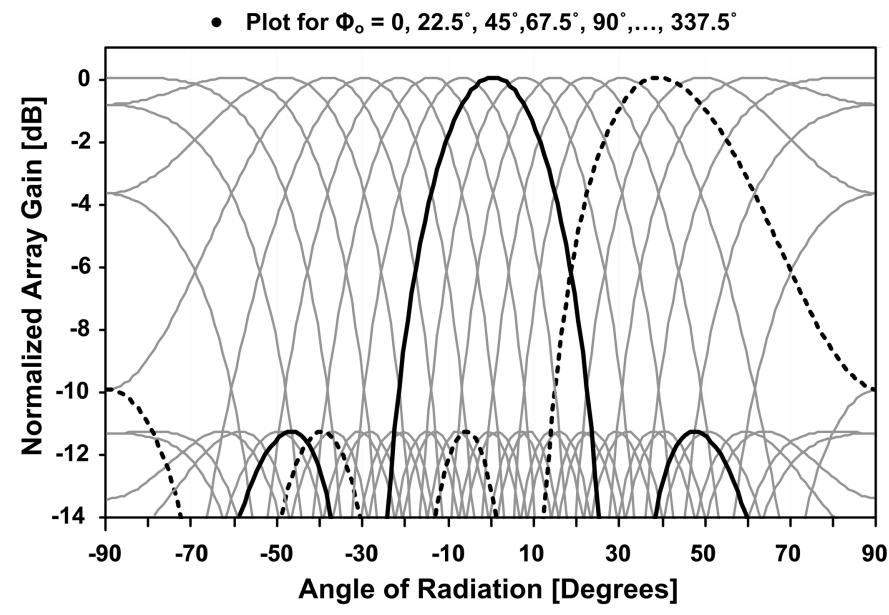

(b)

Fig. 7. Array gain for four-element LO path phase-shifting transmitter. (a) Four-element LO phase-shift phased-array transmitter. (b) Array gain for four-element phased-array transmitter.

shifts of $\phi, 2 \phi$, and $3 \phi$ are implemented in the elements. The angle of radiation $\theta$ is given by

$$
\theta=\sin ^{-1}\left(\frac{\phi c}{\omega_{\mathrm{RF}} d}\right)
$$

where $c$ is the velocity of light and $d$ is the spacing between the antennas. For $d=\lambda_{\mathrm{RF}} / 2$

$$
\theta=\sin ^{-1}\left(\frac{\phi}{\pi}\right)
$$
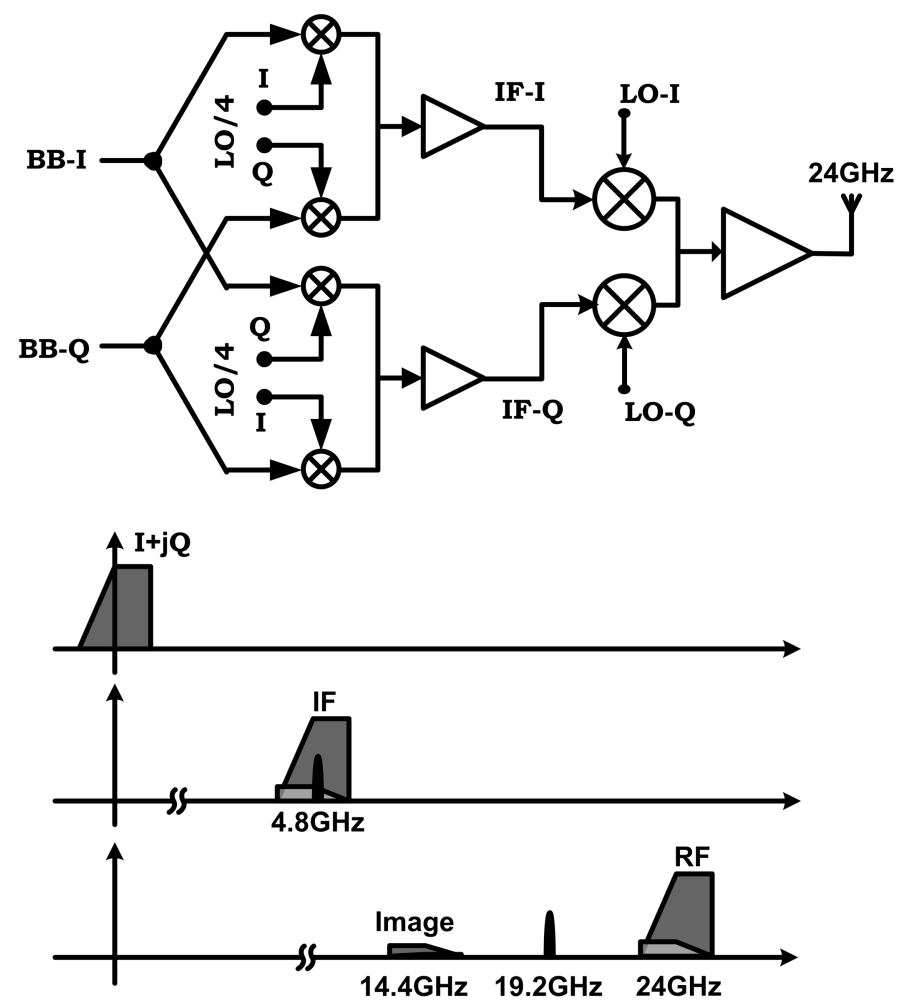

Fig. 8. Equivalent architecture of a single transmitter element and frequency plan.

Fig. 7(b) plots the array gain versus angle of radiation for 16 uniformly spaced values of $\phi$ with a step size of $22.5^{\circ}$. As can be seen from the figure, the phase-shift resolution is sufficient to radiate at all angles at close to peak array gain. The 3-dB beamwidth for radiation that is normal to the axis of the array is $26^{\circ}$ and the beam-steering resolution is $7^{\circ}$. The beamwidth and the beam-steering resolution increase as the angle of radiation becomes more oblique. The array pattern is degraded by mismatches in the signal amplitude and LO phase shift across different elements. For example, in the case of a two-element transmitter, an amplitude mismatch of $3 \mathrm{~dB}$ translates to a peak-tonull ratio of $15.3 \mathrm{~dB}$. The error in the beam direction, due to a given absolute error in LO phase shifting, depends upon the actual beam direction and can be derived from (7).

\section{TRANSMitTER ARCHITECTURE}

In this section, the architecture of the phased-array transmitter is discussed in depth. This is followed by a detailed description of the circuits in the signal path and the LO path.

The four-element fully integrated transmitter has on-chip power amplifiers [19] as well as an integrated frequency synthesizer. Due to concerns related to frequency pulling, direct up-conversion was considered to be unsuitable. A two-step up-conversion architecture was chosen for the transmitter with LO frequencies of 4.8 and $19.2 \mathrm{GHz}$ (Fig. 8). The two LO frequencies are generated by a single synthesizer loop using a divide-by-four.

Quadrature up-conversion was implemented in both stages [20]. The image attenuation of the first up-conversion step depends upon the matching and quadrature accuracy of the 


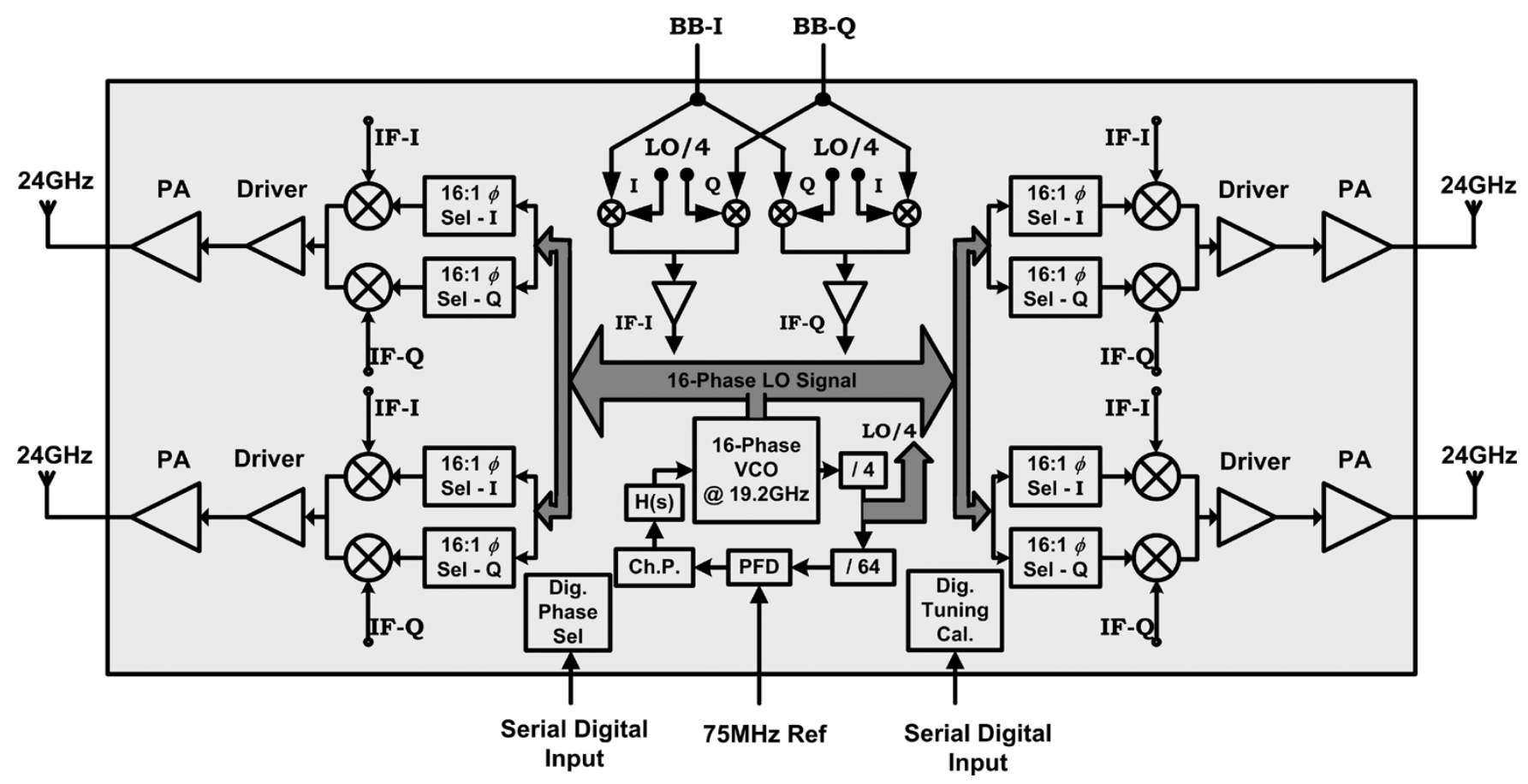

Fig. 9. Architecture and floor plan of 24-GHz four-element phased-array transmitter.

first up-conversion step. The image signal of the second upconversion step falls at $14.4 \mathrm{GHz}$ and is therefore attenuated not only by the quadrature architecture but also by the tuned stages at RF.

Fig. 9 shows the architecture and floor plan of the four-element transmitter [21]. In the signal path, the baseband $I$ and $Q$ signals are up-converted to $4.8 \mathrm{GHz}$ by a pair of quadrature up-conversion mixers. The 4.8-GHz $I$ and $Q$ signals are buffered and provided to the 4.8- to $24-\mathrm{GHz}$ up-conversion mixers in each element. The output of the mixers is amplified and differential to single-ended conversion is performed to drive the on-chip single-ended CMOS power amplifiers that are matched at the output to $50 \Omega$.

In the LO path, the output of the 16-phase 19.2-GHz VCO is provided to the phase selectors in each element. These phase selectors select the right phase of the LO in each element for the desired beam direction. The phase-selection circuitry is controlled by shift registers that can be programmed using a digital serial interface, enabling electronic beam steering. The VCO is part of an on-chip frequency synthesizer that generates the 19.2-GHz LO signals from a 75-MHz reference. A divide-byfour in the synthesizer loop generates the 4.8-GHz LO $I$ and $Q$ signals for the first up-conversion step.

\section{A. Circuits in Signal Path}

1) IF and RF Up-Conversion Mixers: The basebandto-4.8-GHz quadrature mixers common to all elements are Gilbert-type up-conversion mixers. The first up-conversion mixer consumes $3.8 \mathrm{~mA}$ of dc current while the buffers following the mixer consume $4.3 \mathrm{~mA}$. The output of these buffers is distributed to the quadrature up-conversion mixers in each element using a symmetric $\mathrm{H}$-tree structure to ensure good array performance.
Fig. 10(a) shows the schematic of 4.8- to 24-GHz up-conversion mixers in each element. These mixers are essentially Gilbert-type up-conversion mixers with some of the dc current provided by the p-channel field-effect transistors (PFETs) [22], [23]. The current in the IF transconductance part of the mixer $\left(\mathrm{M}_{1}\right.$ and $\left.\mathrm{M}_{2}\right)$ needs to be high to improve mixer linearity and gain. However, the $V_{\mathrm{GS}}$ drop in the switching devices $\left(\mathrm{M}_{3}, \mathrm{M}_{4}\right.$, $\mathrm{M}_{5}$, and $\mathrm{M}_{6}$ ) increases significantly with higher dc current and hence the amplitude of the LO needed to switch these transistors increases. Therefore, to increase the conversion gain at a given LO swing and to reduce the sensitivity of the conversion gain to the LO amplitude, part of the dc current ( $\sim 55 \%)$ is provided by the PFETs $\left(M_{7}\right.$ and $\left.M_{8}\right)$, which reduce the dc current and, consequently, the $V_{\mathrm{GS}}$ drop across the switching devices. Fig. 10(b) plots the simulated conversion gain of the quadrature mixers against $\mathrm{LO}$ amplitude for the same dc current in the IF transconductance part, with and without the PFETs. It can be seen that, with the PFET current sources, the simulated quadrature conversion gain increases from 6 to $9.7 \mathrm{~dB}$ for a $200-\mathrm{mV}$ peak-to-peak LO swing and is less sensitive to LO amplitude. The quadrature mixers draw a total current of $10 \mathrm{~mA}$ from a 2.5-V supply.

2) Tunable Passive Loads at 24 GHz: The cascade of tuned stages in the RF path in each element exacerbates any off-tuning in the passive loads. To avoid the problem of gain loss due to off-tuning, switchable capacitors, controlled by programmable shift registers, were implemented at the output of some of the high-frequency stages [Fig. 11(a)]. In the predriver stage, for example, these capacitors allow the center frequency to be tuned from 23.7 to $26.3 \mathrm{GHz}$, which is sufficient to account for process variations and errors in simulation of passives [Fig. 11(b)].

3) Balun: All the circuits up to and including the $24-\mathrm{GHz}$ PA driver are differential while the PA was designed to be single ended. To avoid power and efficiency loss at the output of the 


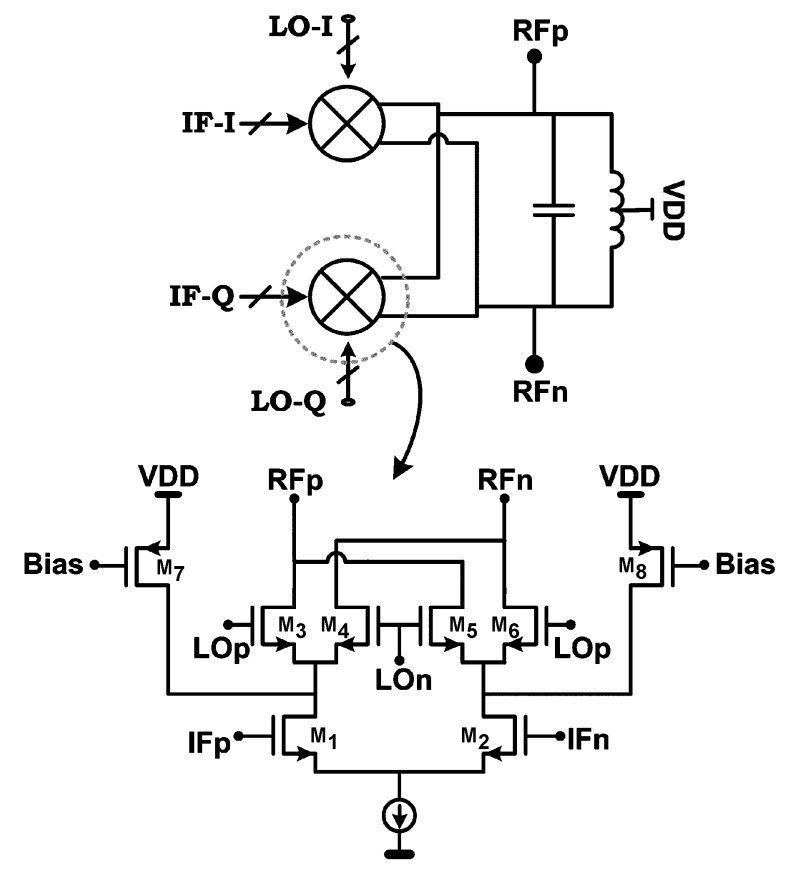

(a)

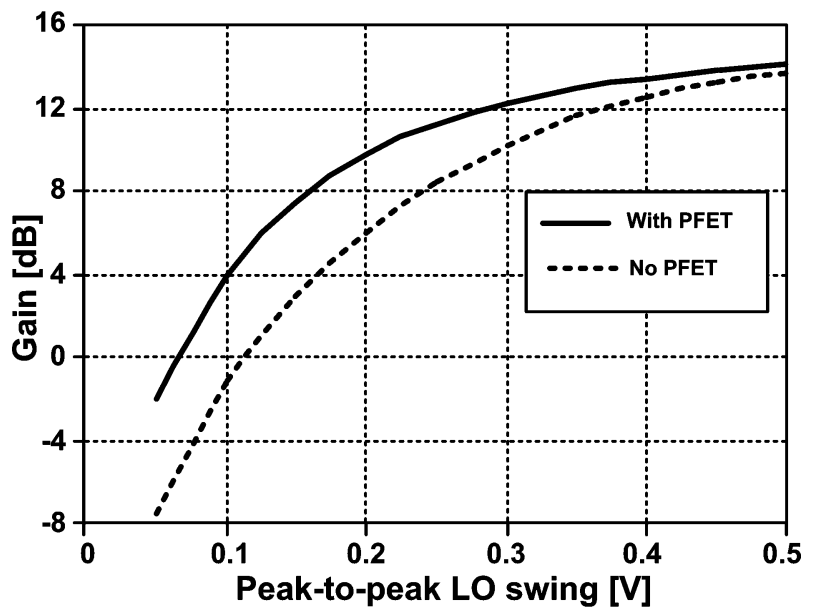

(b)

Fig. 10. RF up-conversion mixers (4.8-24 GHz). (a) Gilbert-type mixer with PFETs for current boosting. (b) Variation of gain with LO amplitude with and without current boosting.

PA, a balanced-unbalanced converter (balun) was placed before the PA. This eliminates the need for an off-chip balun or a differential antenna. As shown in Fig. 12, the balun was realized with a single-turn transformer to minimize substrate loss through capacitive coupling. Electromagnetic simulations show an insertion loss of $1.5 \mathrm{~dB}$ for the balun when input and output parasitic inductances are tuned out with parallel capacitors [24].

4) Power Amplifier (PA): The transmitter contains four on-chip power amplifiers matched at the output to $50 \Omega$. The amplifier, shown in Fig. 13(a), is similar to the stand-alone amplifier reported in [25]. While the input of the stand-alone amplifier is matched to $50 \Omega$, the parasitic inductance of the balun is used to tune out the input capacitance in the first stage of the amplifier integrated in the transmitter. The PA has two gain stages with each gain stage consisting of a cascode transistor pair to ensure stability and increase breakdown voltage. The PA

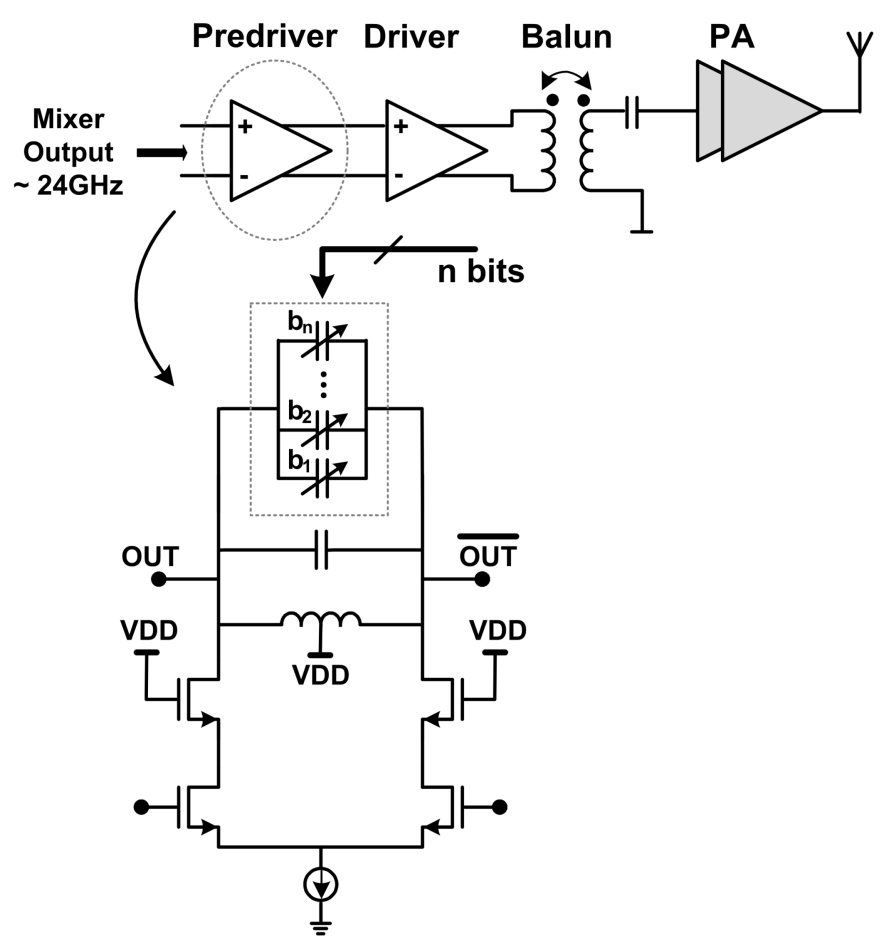

(a)

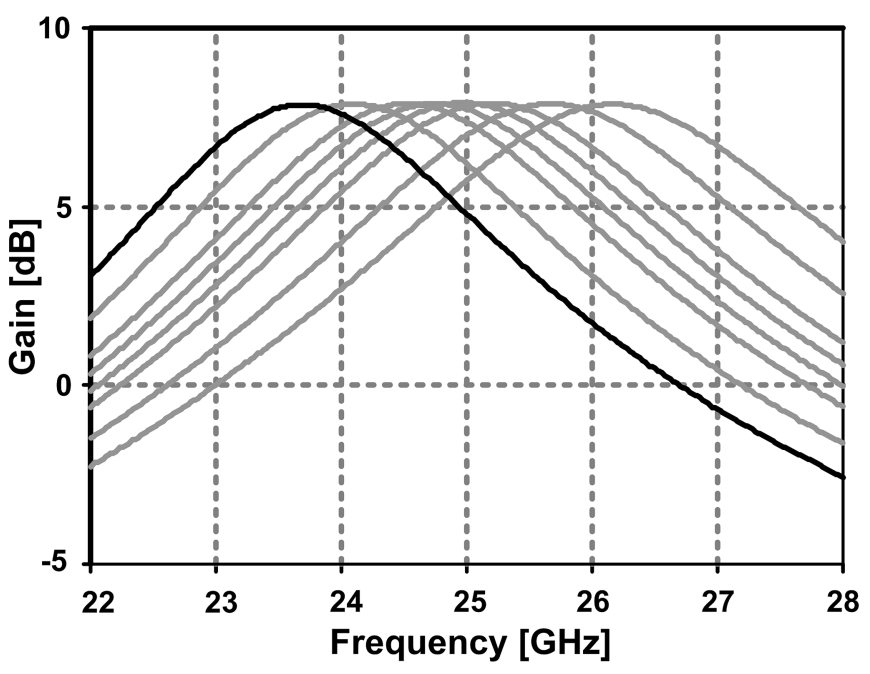

(b)

Fig. 11. Digital tuning calibration in high-frequency stages. (a) Predriver stage with switchable capacitors in load. (b) Simulated change in center frequency with digital tuning control.

is designed to operate in class $\mathrm{AB}$ mode. As the transistor $f_{\max }$ is $65 \mathrm{GHz}$, the harmonic content at the drain of the transistor for the 24-GHz input signal is low. Harmonic-matching-based classes such as class $\mathrm{E}$ and class $\mathrm{F}$ therefore did not increase efficiency significantly in simulation and were not used. The output and interstage matching networks in the PA are realized with the substrate-shielded coplanar waveguide structure shown in Fig. 13(b) to reduce power losses and area [26]. In this structure, the presence of the ground shield beneath the coplanar signal line increases the capacitance per unit length, $C_{u}$. However, as the return current cannot flow through the patterned ground shield, the inductance per unit length, $L_{u}$, remains the same. The simultaneously high $C_{u}$ and $L_{u}$ result 


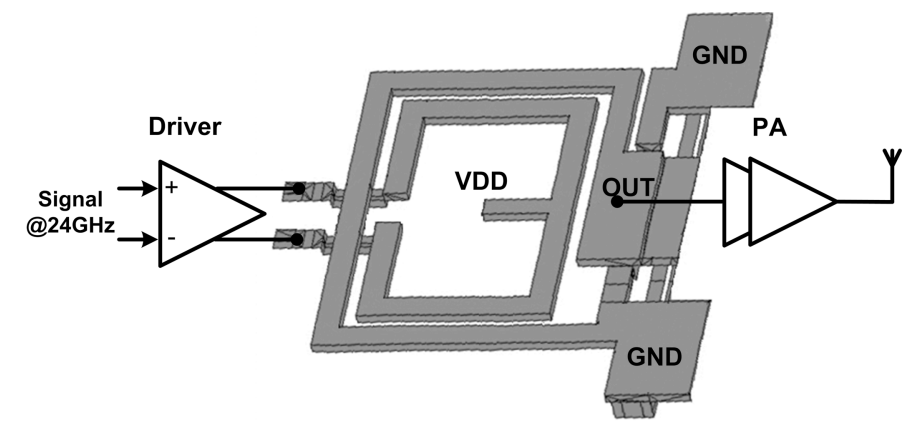

Fig. 12. Balun for differential to single-ended conversion at PA input.

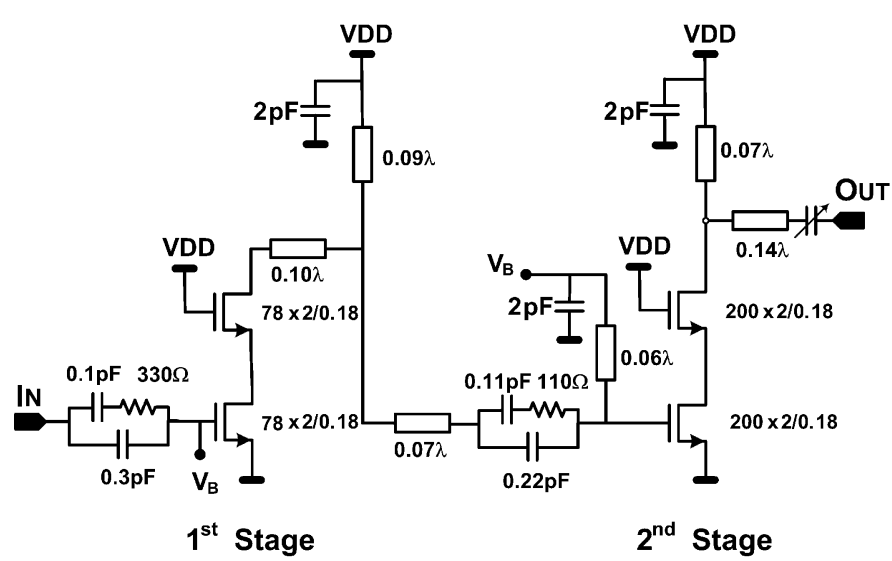

(a)

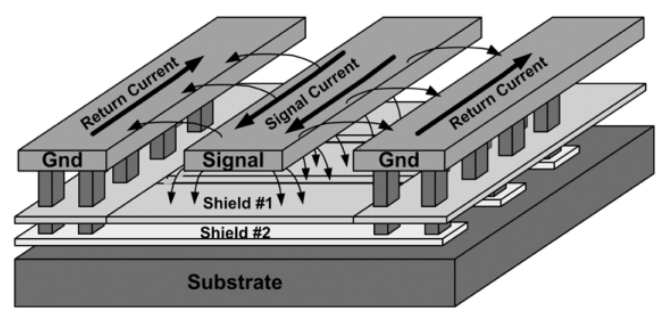

(b)

Fig. 13. On-chip power amplifier. (a) Two-stage on-chip power amplifier. (b) Substrate-shielded coplanar waveguide structure.

in lower wave velocity, leading to more than a factor of two reduction in wavelength at $24 \mathrm{GHz}$ when compared to a standard coplanar waveguide structure in silicon dioxide. Additionally, as the structure is well shielded, the isolation between the power amplifier and other circuits in the transmitter is improved. The low loss per unit length $(1 \mathrm{~dB} / \mathrm{mm})$, improved isolation, and short wavelengths make this structure particularly suitable for integrating multiple power amplifiers on the same die.

Amplifier stability is improved by the RC network at the input of each stage, which guarantees low frequency stability. Further details on the design of the power amplifier and the waveguide structure can be found in [19] and [25].

\section{B. LO Path Circuits}

The multiple-phase VCO, shown in Fig. 14, is at the heart of the LO phase-shifting architecture adopted in this paper. The 19.2-GHz CMOS VCO consists of eight differential amplifiers connected together in a ring structure and is similar to the design in [14]. As the ring is closed by flipping the inputs of the last

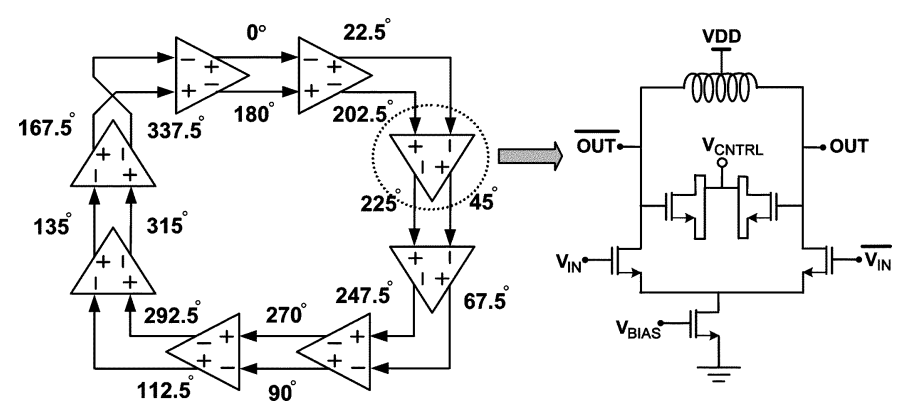

Fig. 14. 19.2-GHz 16-phase CMOS VCO.

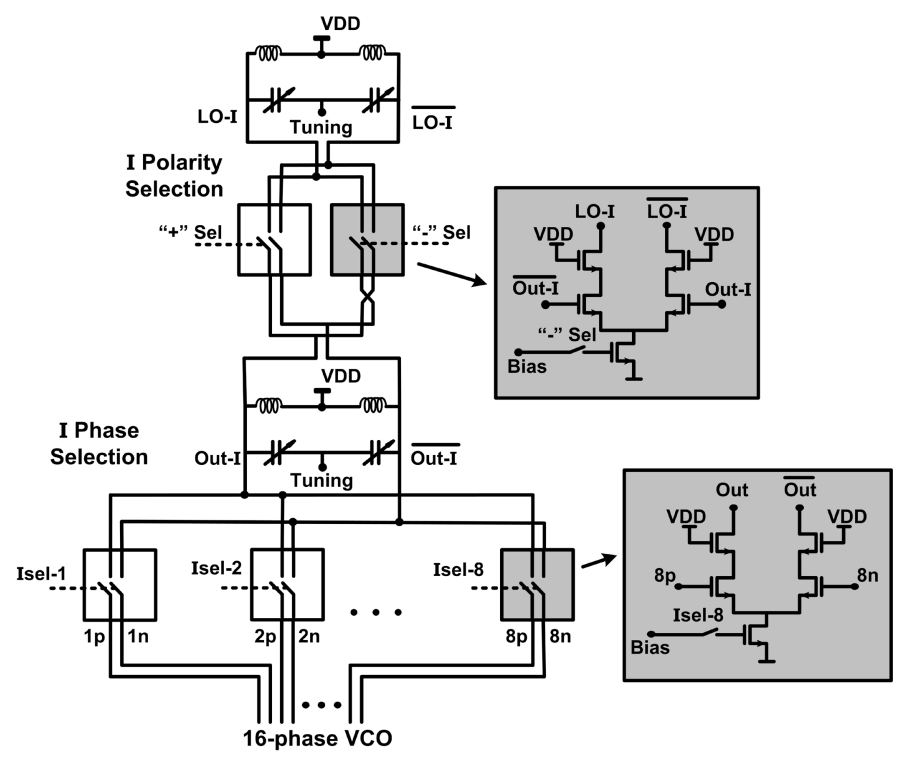

Fig. 15. Two-stage phase selector.

amplifier, the VCO is capable of generating 16 equally spaced phases of the LO with a step size of $22.5^{\circ}$ [27]. As previously discussed, this step size is sufficient for a four-element phasedarray system.

The outputs of the VCO have to be provided to the phase selectors in each element in a symmetric fashion as any asymmetry in this distribution leads to an error in the phase shift causing degradation of the array pattern. Therefore, a symmetric H-tree structure, using the top two thick metal layers, is used to distribute the multiple VCO outputs.

The phase selectors for the $I$ and $Q$ LO phases in each element work in two stages, with the first stage selecting the right differential pair and the second stage determining the polarity. The eight differential outputs of the VCO are provided to two sets of eight differential pairs that comprise the first stages of the $I$ and $Q$ phase selectors. The differential outputs are also provided to a dummy set of differential pairs to ensure that the VCO buffers see a constant load. Fig. 15 shows the phase-selection circuitry that determines the phase for the $I$ signal to the mixer LO ports. An identical circuit selects the phase for the $Q$ signal independently. As shown in Fig. 15, the tail current of each differential pair in the first stage is controlled by a shift register. By turning on the right differential pair in the first stage, the VCO differential pair outputs corresponding to desired phase for the $I$ signal can be selected. The second stage of the phase selectors 


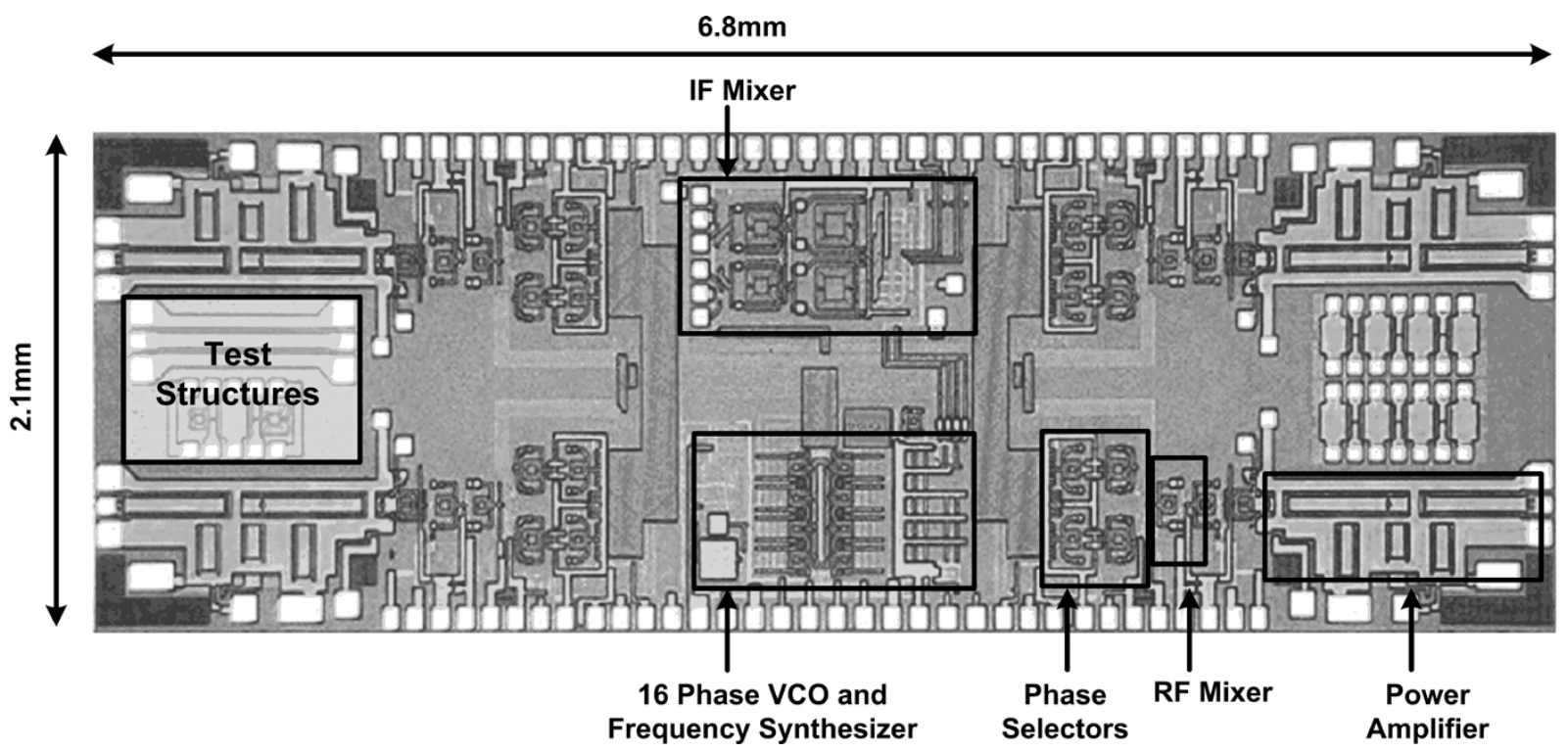

Fig. 16. Die micrograph of 24-GHz four-element phased-array transmitter.

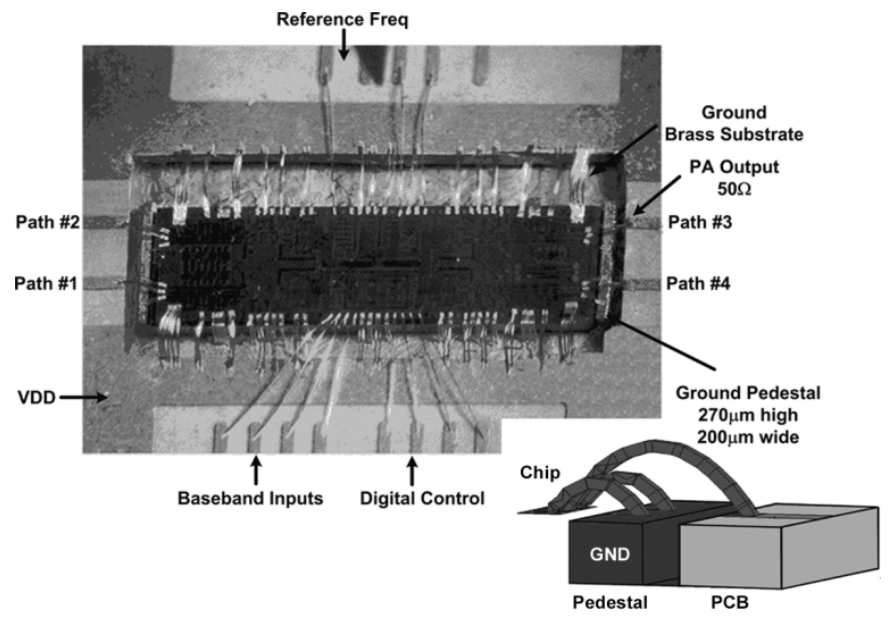

Fig. 17. Measurement setup to characterize transmitter performance.

consists of a set of two differential pairs with tail current sources that are also controlled by the shift registers (Fig. 15). The inputs to these differential pairs are anti-phase with respect to each other. Thus, the output can either be in-phase or anti-phase with respect to the input. In the dummy set, the tail current sources of the differential pairs is controlled by bits complementary to the LO $I$ and $Q$ phase-selection bits to ensure constant loading of the VCO buffers. The eight differential pairs (six dummy, one $I$, and one $Q$ ) that are turned on for any given phase shift draw a total of $16 \mathrm{~mA}$ and the differential pairs in the second stage draw $3.1 \mathrm{~mA}$ each. While independent $I$ and $Q$ LO phase selection provides flexibility to account for phase distribution and device mismatches, in practice the LO distribution and matching was sufficient to render independent selection superfluous.

Interpolation of the raw 16 phases of the VCO is possible by selecting more than one differential pair in the first stage of the phase selectors. (The two-stage phase selection procedure limits the phase shifts that can be generated by using this interpolation method.) For example, when the differential pairs corresponding to $0^{\circ}$ phase and $22.5^{\circ}$ phase are enabled, the output has a phase of $11.25^{\circ}$.

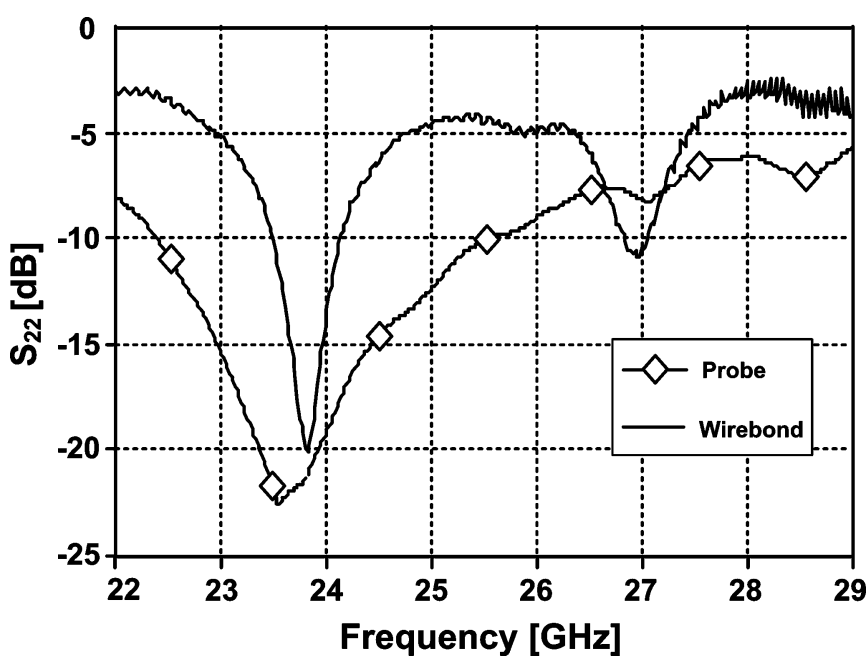

Fig. 18. Output matching with probe-based testing and with wirebonds to PCB.

\section{EXPERIMENTAL RESULTS}

The phased-array transmitter has four elements and is implemented using 0.18- $\mu \mathrm{m}$ CMOS transistors in a BiCMOS process [28]. The $f_{T}$ of the NMOS transistors in the process is $65 \mathrm{GHz}$. The process offers five metal layers with the thickness of the top two metal layers being $4 \mu \mathrm{m}$ and $1.25 \mu \mathrm{m}$, respectively. Fig. 16 shows a die photograph of the transmitter which occupies $6.8 \mathrm{~mm} \times 2.1 \mathrm{~mm}$ of die area.

A high-frequency printed circuit board (PCB) measurement setup has been designed to characterize complete system performance. Some of the packaging parasitics such as wirebond inductance have been taken into account during circuit design. Fig. 17 shows a close-up of the measurement setup for the transmitter chip. The PCB is a high-frequency laminate that is compatible with planar antenna design and is supported by a brass substrate that acts as the ground. Two ground pedestals are milled on the brass substrate and the chip is mounted on the substrate, between the pedestals, using silver epoxy. By wirebonding the PA ground pads onto the pedestals, the length 


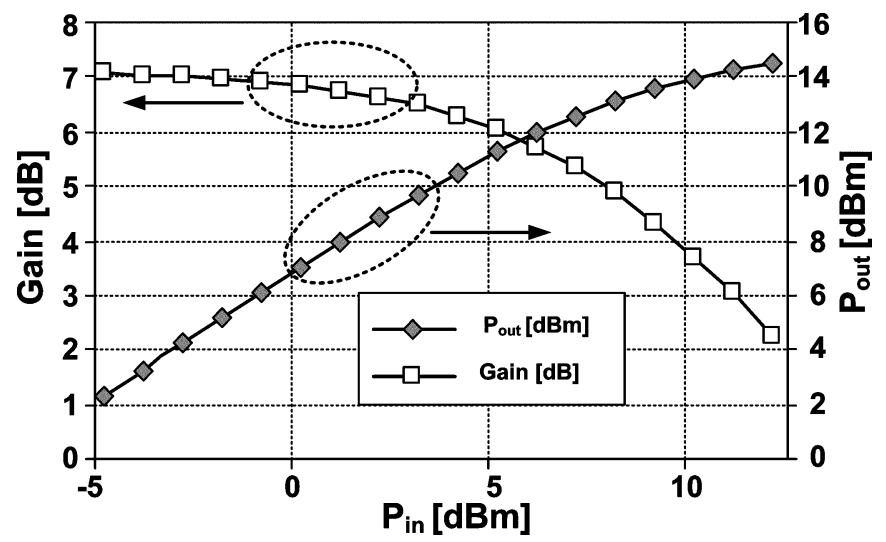

Fig. 19. Performance of on-chip 24-GHz CMOS power amplifier.

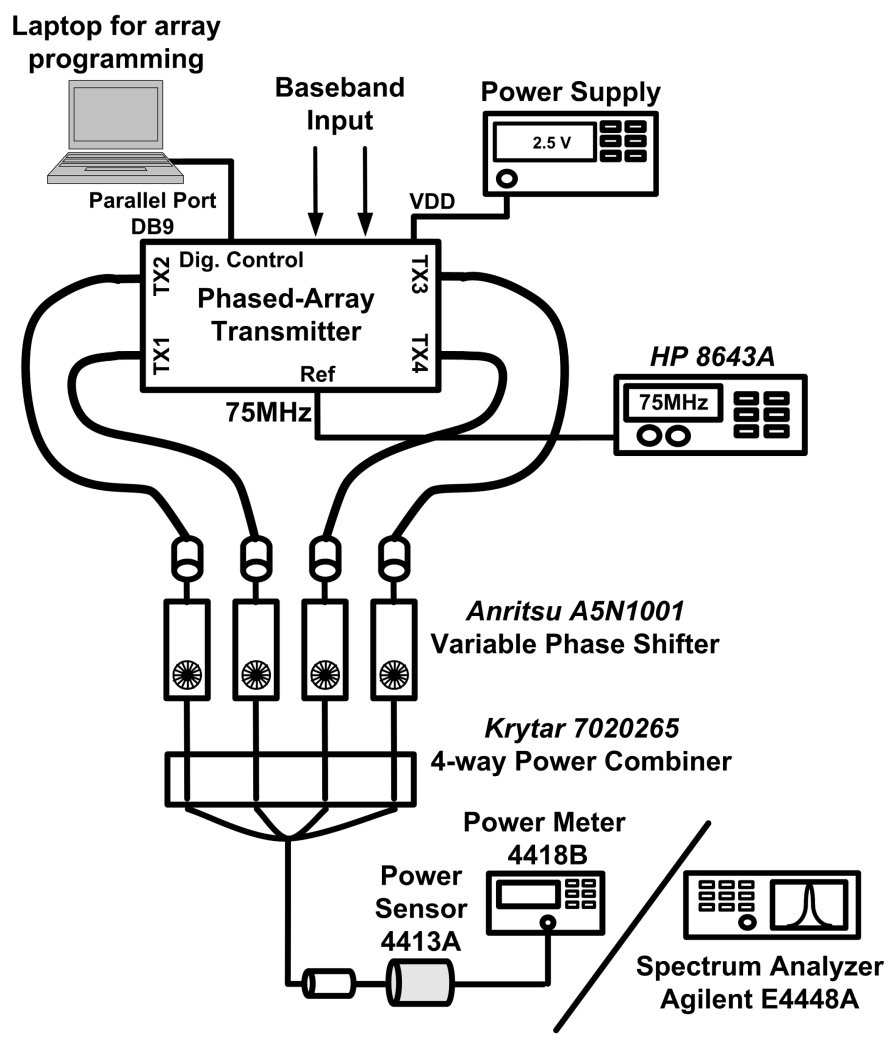

Fig. 20. Array measurement setup.

and therefore the inductance of these wirebonds are reduced. The height of the PCB (10-mil dielectric thickness) is chosen to be close to the height of the chip $(\sim 350 \mu \mathrm{m})$ to reduce the length of the wirebonds to traces on the PCB. Fig. 18 shows the output matching of a single element of the transmitter when measured by probing and by wirebonding the output to the PCB. Though the match with wirebonds is more narrowband, the output matching is better than $10 \mathrm{~dB}$ from 23.4 to $24.1 \mathrm{GHz}$. A broader frequency range for matching can be achieved by using flip-chip-based packaging techniques.

Fig. 19 summarizes the performance of the on-chip power amplifiers, which are capable of generating up to $14.5 \mathrm{dBm}$ of output power at $24 \mathrm{GHz}$ with an output-referred $1-\mathrm{dB}$ compression point of $11 \mathrm{dBm}$. The coupling between multiple power amplifiers on the same die is a concern in an integrated phased-array system. The physical distance $(\sim 1 \mathrm{~mm})$ between
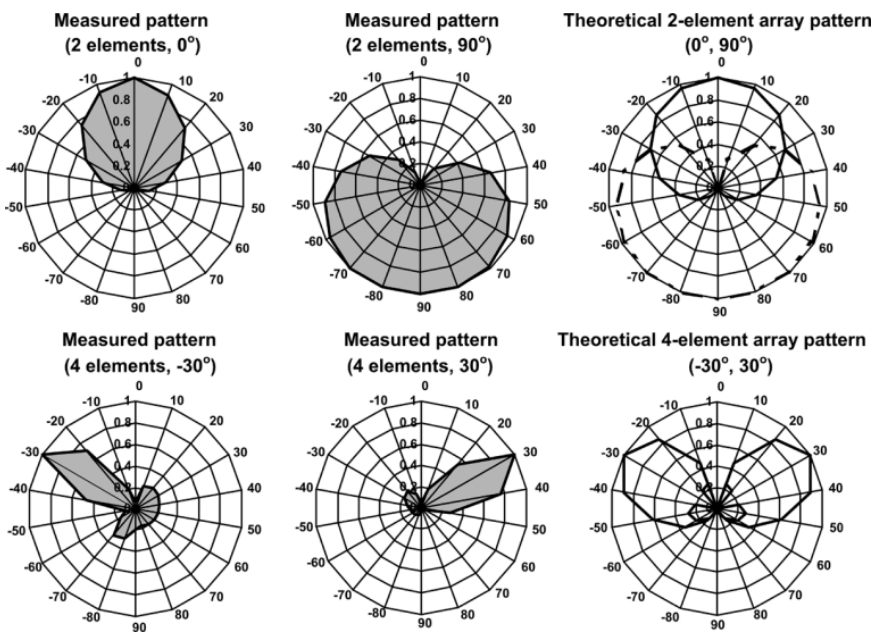

Fig. 21. Comparison of theoretical and measured array pattern with two elements and with four elements active.

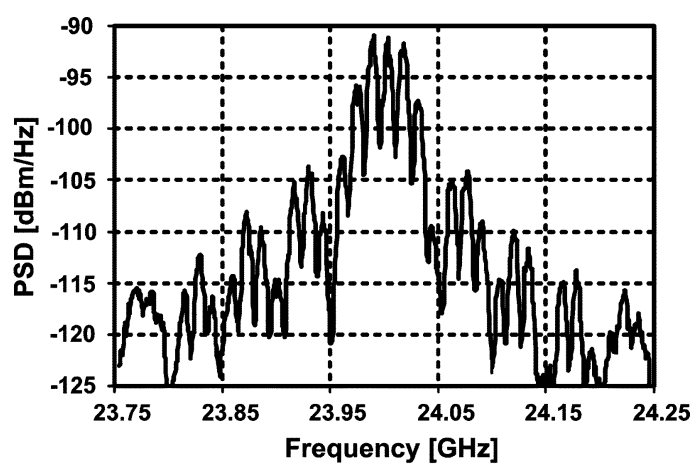

(a)

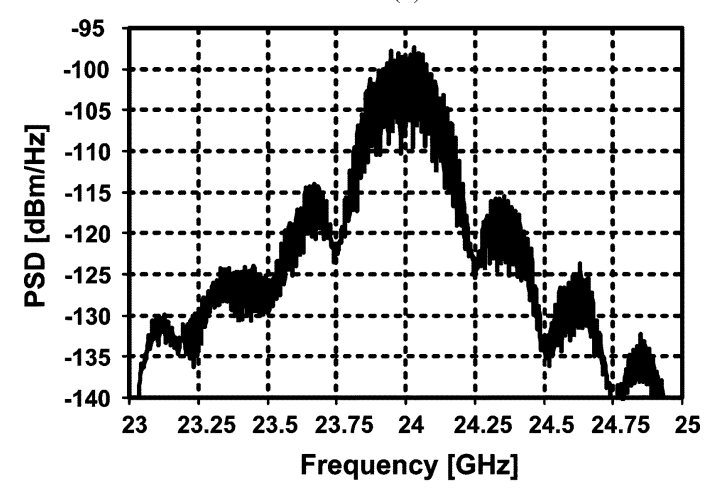

(b)

Fig. 22. Output spectrum of transmitter for 100- and 500-Mb/s QPSK input. (a) Output spectrum for $100-\mathrm{Mb} / \mathrm{s}$ QPSK input. (b) Output spectrum for 500-Mb/s QPSK input.

the power amplifiers and the use of a shielded transmission line in matching networks improve the isolation in this work. In order to measure the isolation between elements, three of the elements were deactivated by switching off all the LO phases in the phase selectors in those elements. The isolation was determined by comparing the power at the output of the active element with the output power of the three inactive elements. The worst case isolation (i.e., between two adjacent elements) is measured to be $28 \mathrm{~dB}$.

The image rejection of the first up-conversion stage, which depends upon the quadrature matching of the mixers and the first 


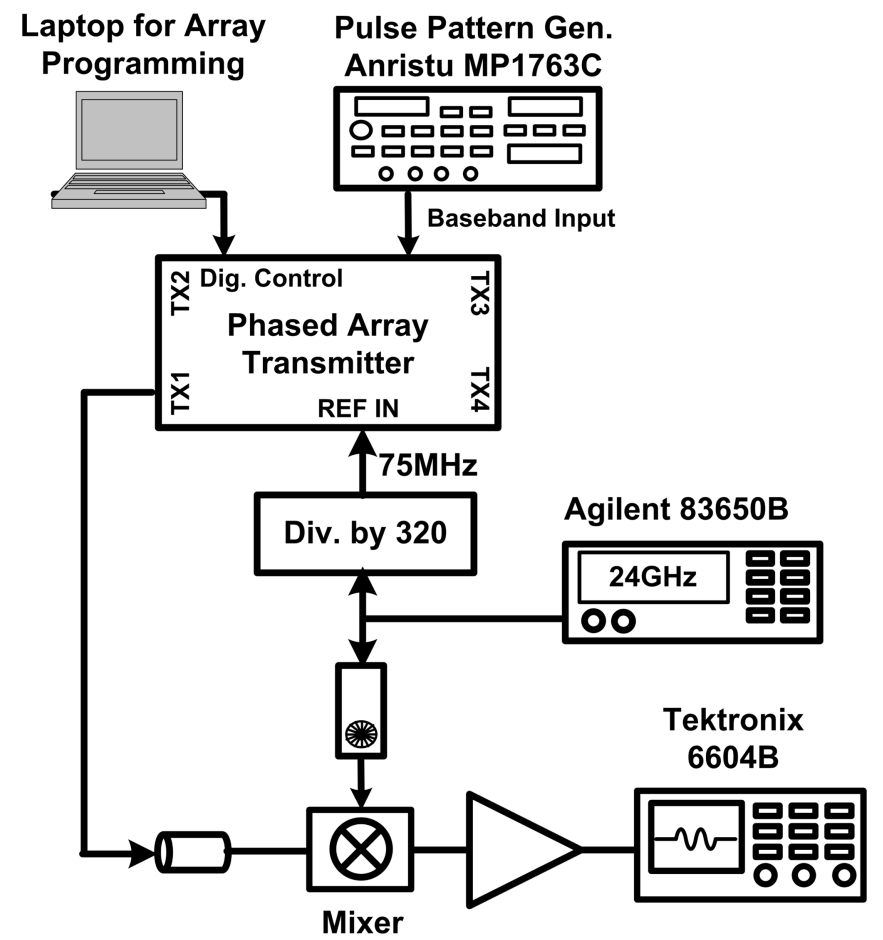

Fig. 23. Setup for direct down-conversion of 24-GHz transmitter output.

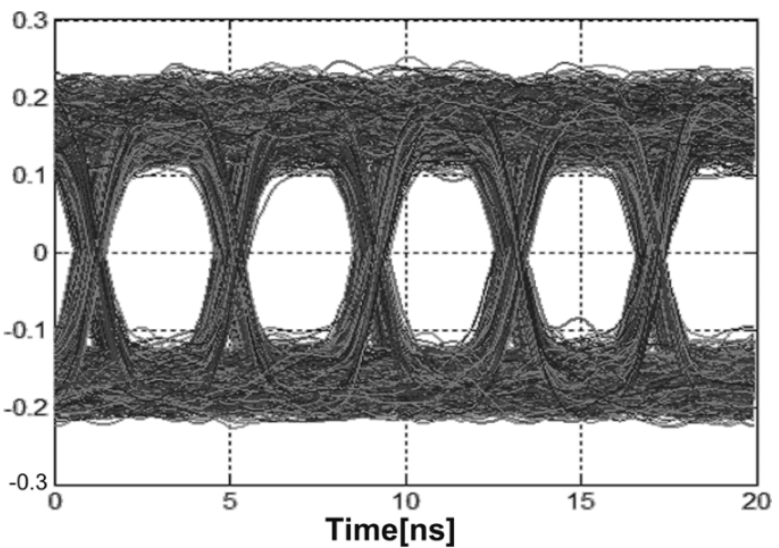

(a)

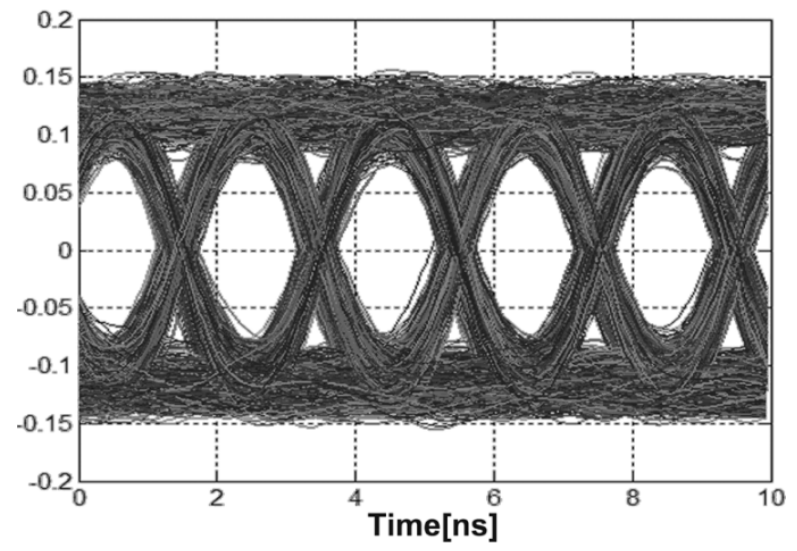

(b)

Fig. 24. Eye diagram of the down-converted transmitter output for 250- and $500-\mathrm{Mb} / \mathrm{s}$ BPSK input. (a) Single-channel $250 \mathrm{Mb} / \mathrm{s}$ (only to $I$ channel): $\mathrm{EVM}=9.7 \%$. (b) Single-channel $500 \mathrm{Mb} / \mathrm{s}$ (only to $I$ channel): $\mathrm{EVM}=9.8 \%$.
TABLE I

TRANSMITTER PERFORMANCE SUMMARY

On-Chip CMOS Power Amplifier Performance

Maximum Saturated Output Power

$+14 \mathrm{dBm}$

Current Consumption @ 2.5V

$68 \mathrm{~mA}$

Output Match @ 24GHz

$-20 \mathrm{~dB}$

Equivalent 4-element EIRP

Output Referred 1dB compression point

$+26 \mathrm{dBm}$

Peak PAE

Output $3^{\text {rd }}$-order Intercept Point (OIP3)

$11 \mathrm{dBm}$

$6.5 \%$

$14 \mathrm{dBm}$

\section{Phased Array Performance}

Peak-to-Null Ratio for 4-element Array

$>23 \mathrm{~dB}$

Beam Steering Resolution (for normal radiation)

$<10^{\circ}$

3dB Array Beam-Width @ 30 Radiation Angle

$17^{\circ}$

Isolation between Paths (including wire bonds)

$>28 \mathrm{~dB}$

Image Signal Attenuation

First upconversion

$>24 \mathrm{~dB}$

Second upconversion (image @ 14.4GHz)

$>43 \mathrm{~dB}$

Transmit 3dB Bandwidth

$>400 \mathrm{MHz}$

\section{Current Consumption @ 2.5V}

Signal Path (per element)

Phase Selector (per element)

16-Phase Frequency Synthesizer

$26 \mathrm{~mA}$

Total ( including IF stage and VCO buffers)

$34 \mathrm{~mA}$

$180 \mathrm{~mA}$

$788 \mathrm{~mA}$

Device Technology

$0.18 \mu \mathrm{m}$ CMOS

Die Area

$6.8 \mathrm{~mm} \times 2.1 \mathrm{~mm}$

$\mathrm{LO}$, is $24 \mathrm{~dB}$. The image signal of the second up-conversion step, which falls at $14.4 \mathrm{GHz}$, is found to be attenuated by $43 \mathrm{~dB}$ due to the additional attenuation provided by the tuned stages at RF.

To measure the performance of the transmitter alone, without antenna nonidealities such as coupling, the different propagation delays for each element for each direction of radiation have to be replicated. This is done by connecting the output of each element to variable phase shifters (Fig. 20). By varying the relative phase shift in the external phase shifters, the propagation delays for each beam direction can be emulated. The output of the phase shifters is combined and measured using a spectrum analyzer or a power meter. Fig. 21 shows the measured performance of the transmitter with two elements active and with all four elements active. When compared to theory, these results demonstrate the proper functioning of the phased-array transmitter. The worst-case peak-to-null ratio with all four elements active is $23 \mathrm{~dB}$.

While the PA has a bandwidth of $3.1 \mathrm{GHz}$, the bandwidth of the entire transmitter is constrained by the bandwidth of the IF stages and also by the cascade of tuned stages at IF and RF. Fig. 22, which shows the measured spectrum of the transmitter when a $100-$ and $500-\mathrm{Mb} / \mathrm{s}$ QPSK signal is provided at baseband, indicates that the transmitter is capable of supporting high data rates. To measure the performance of the transmitter for high data rate input, an external direct down-conversion receiver was assembled, consisting of a passive mixer followed 
by an amplifier (Fig. 23). For this measurement, the LO signal that is used for down-conversion needs to be locked to the carrier signal of the transmitter. Therefore, the 24-GHz LO signal to the external mixer is divided by 320 to generate the $75-\mathrm{MHz}$ reference for the on-chip frequency synthesizer. The baseband input was provided to one channel using a pseudorandom bit pattern generator and no pulse shaping was done at the input to minimize intersymbol interference (ISI). Fig. 24 plots the eye diagram for the down-converted baseband filtered output for the 250- and 500-Mb/s BPSK signal. The measured EVM did not increase significantly with an increase in data rate, indicating that it is dominated by the noise in the external down-conversion setup.

The entire system with four on-chip power amplifiers draws $788 \mathrm{~mA}$ from a 2.5-V supply. The measured performance of the chip is summarized in Table I.

\section{CONCLUSION}

In this paper, the first fully integrated phased-array transmitter has been demonstrated using $0.18-\mu \mathrm{m}$ CMOS transistors, proving the feasibility of high-frequency integrated phased-array systems on silicon-based processes. The tradeoffs of high-frequency wireless communication are discussed. The limitations of phase-shifting architectures are explored and methods to overcome them are suggested. The 4-bit local oscillator (LO) path phase-shifting approach adopted in the transmitter has better than $10^{\circ}$ beam-steering resolution for radiation normal to the array. Each on-chip PA is capable of generating up to $14.5 \mathrm{dBm}$ of output power, translating to an effective isotropic radiated power (EIRP) of $26.5 \mathrm{dBm}$. The transmitter is capable of supporting data rates in excess of $500 \mathrm{Mb} / \mathrm{s}$ and is well suited for $24-\mathrm{GHz}$ wireless links.

\section{ACKNOWLEDGMENT}

The authors would like to thank X. Guan and H. Hashemi for their technical contributions and discussions and B. Analui, A. Babakhani, E. Afshari, Prof. David Rutledge, and the anonymous reviewers for their suggestions and feedback. They would also like to acknowledge IBM Corporation for chip fabrication. N. Wadefalk and A. Shen assisted in printed circuit board preparation and packaging. The authors also thank the Lee Center for Advanced Networking for support.

\section{REFERENCES}

[1] R. C. Hansen, Ed., Significant Phased Array Papers. Norwood, MA: Artech House, 1973.

[2] D. Parker and D. C. Zimmermann, "Phased arrays—Part I: Theory and architectures," IEEE Trans. Microw. Theory Tech., vol. 50, no. 3, pp. 678-687, Mar. 2002.

[3] — , "Phased-arrays_-Part II: Implementations, applications, and future trends," IEEE Trans. Microw. Theory Tech., vol. 50, no. 3, pp. 688-698, Mar. 2002.

[4] Y. Akamine et al., "A loop-bandwidth calibration system for fractional- $N$ synthesizer and $\Delta \Sigma$ PLL transmitter," in IEEE Int. Solid-State Circuits Conf. (ISSCC) Dig. Tech. Papers, vol. 48, San Francisco, CA, Feb. 2005, pp. 314-315.

[5] R. B. Staszewski et al., "All-digital PLL and GSM/EDGE transmitter in $90 \mathrm{~nm}$ CMOS," in IEEE Int. Solid-State Circuits Conf. (ISSCC) Dig. of Tech. Papers, vol. 48, San Francisco, CA, Feb. 2005, pp. 316-317.
[6] X. Guan, H. Hashemi, and A. Hajimiri, "A fully integrated 24-GHz eight-element phased-array receiver in silicon," IEEE J. Solid-State Circuits, vol. 39, no. 12, pp. 2311-2320, Dec. 2004.

[7] J. Paramesh, R. Bishop, K. Soumyanath, and D. Allstot, "A 1.4 V 5 $\mathrm{GHz}$ four-antenna Cartesian-combining receiver in $90 \mathrm{~nm}$ CMOS for beamforming and spatial diversity applications," in IEEE Int. Solid-State Circuits Conf. (ISSCC) Dig. Tech. Papers, vol. 48, San Francisco, CA, Feb. 2005, pp. 210-211.

[8] Federal Communications Commission, FCC 02-04, Section 15.515.15.249.

[9] D. Lu, D. B. Rutledge, M. Kovacevic, and J. Hacker, "A 24 GHz patch array with a power amplifier/low-noise amplifier MMIC," Int. J. Infrared Millim. Waves, vol. 23, pp. 693-704, May 2002.

[10] D. B. Rutledge, The Electronics of Radio. New York: Cambridge Univ. Press, 1999.

[11] C. A. Balanis, Antenna Theory: Analysis and Design, 2nd ed. New York: Wiley, 1997.

[12] C. E. Shannon, "A mathematical theory of communications," Bell Syst. Tech. J., vol. 27, no. 3, pp. 379-423 and 623-656, 1948.

[13] T. Lee, The Design of CMOS Radio-Frequency Integrated Circuits, 2nd ed. New York: Cambridge Univ. Press, 2004.

[14] H. Hashemi, X. Guan, A. Komijani, and A. Hajimiri, "A 24-GHz SiGe phased-array receiver-LO phase shifting approach," IEEE Trans. Microw. Theory Tech., vol. 53, no. 2, pp. 614-626, Feb. 2005.

[15] H. Zarei and D. J. Allstot, "A low-loss phase shifter in $180 \mathrm{~nm} \mathrm{CMOS}$ for multiple-antenna receivers," in IEEE Int. Solid-State Circuits Conf. (ISSCC) Dig. Tech. Papers, vol. 47, San Francisco, CA, Feb. 2004, pp. 392-534.

[16] M. Chua and K. Martin, "A $1 \mathrm{GHz}$ programmable analog phase shifter for adaptive antennas," in Proc. IEEE Custom Integrated Circuits Conf., Santa Clara, CA, May 1998, pp. 11-14.

[17] A. Hajimiri, A. Komijani, A. Natarajan, X. Guan, and H. Hashemi, "Phased-array systems in silicon," IEEE Commun. Mag., vol. 42, no. 8, pp. 122-130, Aug. 2004.

[18] G. L. Stuber, J. R. Barry, S. W. McLaughlin, Y. Li, M. A. Ingram, and T G. Pratt, "Broadband MIMO-OFDM wireless communications," Proc. IEEE, vol. 92, no. 2, pp. 271-294, Feb. 2004.

[19] A. Komijani, A. Natarajan, and A. Hajimiri, "A $24 \mathrm{GHz},+14.5 \mathrm{dBm}$ fully-integrated power amplifier in $0.18 \mu \mathrm{m}$ CMOS," IEEE J. Solid-State Circuits, vol. 40, no. 9, pp. 1901-1908, Sep. 2005.

[20] D. K. Weaver, "A third method of generation and detection of single-sideband signals," Proc. IRE, vol. 44, no. 44, pp. 1703-1705, Dec. 1956.

[21] A. Natarajan, A. Komijani, and A. Hajimiri, "A $24 \mathrm{GHz}$ phased-array transmitter in $0.18 \mu \mathrm{m} \mathrm{CMOS,"} \mathrm{in} \mathrm{IEEE} \mathrm{Int.} \mathrm{Solid-State} \mathrm{Circuits} \mathrm{Conf.}$ (ISSCC) Dig. Tech. Papers, vol. 48, San Francisco, CA, Feb. 2005, pp. 212-213.

[22] J. Jussila, J. Ryyanen, K. Kivakas, L. Sumanen, A. Parssinen, and K. Haionen, "A $22 \mathrm{~mA} 3.7 \mathrm{~dB} \mathrm{NF}$ direct conversion receiver for 3 G WCDMA," in IEEE Int. Solid-State Circuits Conf. (ISSCC) Dig. Tech. Papers, vol. 44, San Francisco, CA, Feb. 2001, pp. 284-285.

[23] Z. Zhang, Z. Chen, L. Tsui, and J. Lau, "930 MHz CMOS DC-offsetfree direct-conversion 4-FSK receiver," in IEEE Int. Solid-State Circuits Conf. (ISSCC) Dig. Tech. Papers, vol. 44, San Francisco, CA, Feb. 2001, pp. 290-291.

[24] IE3D Electromagnetic Simulation User Manual, Release 8, Zeland Software Inc., Zeland, CA, 2001.

[25] A. Komijani and A. Hajimiri, "A $24 \mathrm{GHz},+14.5 \mathrm{dBm}$ fully-integrated power amplifier in $0.18 \mu \mathrm{m} \mathrm{CMOS,"} \mathrm{in} \mathrm{Proc.} \mathrm{Custom} \mathrm{Integrated} \mathrm{Circuits}$ Conf., Orlando, FL, Oct. 2004, pp. 561-564.

[26] T. S. D. Cheung et al., "On-chip interconnect for mm-wave applications using an all-copper technology and wavelength reduction," in IEEE Int Solid-State Circuits Conf. (ISSCC) Dig. Tech. Papers, vol. 46, San Francisco, CA, Feb. 2003, pp. 396-397.

[27] J. Savoj and B. Razavi, "A 10-Gb/s CMOS clock and data recovery circuit with a half-rate binary phase-frequency detector," IEEE J. SolidState Circuits, vol. 38, no. 1, pp. 13-21, Jan. 2003.

[28] A. Joseph et al., "A $0.18 \mu \mathrm{m}$ BiCMOS technology featuring 120/100 $\mathrm{GHz}$ (ft/fmax) HBT and ASIC-compatible CMOS using copper interconnect," in Proc. Bipolar/BiCMOS Circuits and Technology Meeting (BCTM), Minneapolis, MN, 2001, pp. 143-146. 


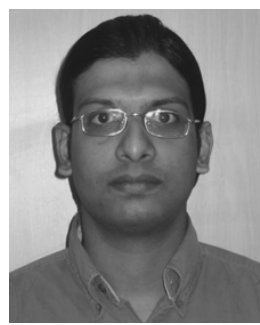

Arun Natarajan (S'02) received the B.Tech. degree in electrical engineering from the Indian Institute of Technology, Madras, India, and the M.S. degree from the California Institute of Technology (Caltech), Pasadena, CA, where he is currently working toward the Ph.D. degree.

In the summer of 2005, he was a Design Engineer with the IBM T. J. Watson Research Center, Yorktown Heights, NY, where he worked on high-frequency systems. His research interests include design of integrated high-frequency circuits, wireless transceivers, and modeling of parasitic coupling in integrated circuits.

Mr. Natarajan was the recipient of the Caltech Atwood Fellowship in 2001, the Analog Devices Outstanding Student IC Designer Award in 2004, and the IBM Research Fellowship in 2005.

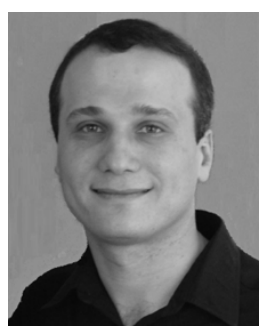

Abbas Komijani (S'98) received the B.S. and M.S. degrees in electronics engineering from the Sharif University of Technology, Tehran, Iran, in 1995 and 1997, respectively. He is currently working toward the Ph.D. degree at the California Institute of Technology (Caltech), Pasadena, CA.

From 1997 to 1999, he was a Senior Design Engineer with Emad Semiconductors, Tehran, where he worked on CMOS chipsets for voiceband applications. From 1999 to 2000, he was a Senior Design Engineer with Valence Semiconductors, Irvine, CA, where he was involved with data converters for voice over Internet Protocol (VoIP) applications. His research interests include high-frequency power amplifiers, wireless transceivers, phased-array architectures, and delta-sigma data converters.

Mr. Komijani was the recipient of the Silver Medal in the National Mathematics Olympiad in 1991, the Custom Integrated Circuits Conference 2004 Best Student Paper Award, Caltech's Atwood Fellowship in 2000, and the Analog Devices Outstanding Student Designer Award in 2005.

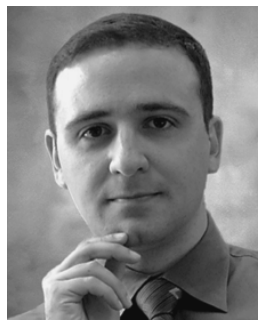

Ali Hajimiri (S'94-M'99) received the B.S. degree in electronics engineering from the Sharif University of Technology, Tehran, Iran, and the M.S. and Ph.D. degrees in electrical engineering from Stanford University, Stanford, CA.

From 1993 to 1994, he was a Design Engineer with Philips Semiconductors, where he was involved with a BiCMOS chipset for GSM and cellular units. In 1995, he was with Sun Microsystems, where he worked on the UltraSPARC microprocessor's cache RAM design methodology. During the summer of 1997, he was with Lucent Technologies, Bell Laboratories, Murray Hill, NJ, where he investigated low-phase-noise integrated oscillators. In 1998, he joined the Faculty at the California Institute of Technology (Caltech), Pasadena, CA, where he is an Associate Professor of electrical engineering and the Director of Microelectronics and Noise Laboratories. He is a cofounder of Axiom Microdevices Inc. His research interests are high-speed and RF integrated circuits. He is the coauthor of The Design of Low Noise Oscillators (Kluwer, 1999) and holds several U.S. and European patents.

Dr. Hajimiri is an Associate Editor of the IEEE Journal of SOLID-STATE CIRCUITS and a Member of the Technical Program Committee of the International Solid-State Circuits Conference (ISSCC). He is a member of the SolidState Circuits Society AdCom. He has also served as an Associate Editor of IEEE TRANSACTIONS ON CIRCUITS AND SYSTEMS Part II, a Member of the Technical Program Committees of the International Conference on Computer Aided Design (ICCAD), Guest Editor of the IEEE TRAnSACTIONS ON MicrowaVE THEORY AND TECHNIQUES, and the Guest Editorial Board of the Transactions of Institute of Electronics, Information and Communication Engineers (IEICE) of Japan. He is on the top 100 innovators (TR100) list. He was the Gold Medal Winner of the National Physics Competition and the Bronze Medal Winner of the 21st International Physics Olympiad, Groningen, Netherlands. He was a corecipient of the ISSCC 1998 Jack Kilby Outstanding Paper Award and a three-time winner of the IBM Faculty Partnership Award as well as the National Science Foundation CAREER award. 